\title{
Knots, links and branes at large $\mathbf{N}$
}

\section{Citation}

Labastida, José M.F, Marcos Mariño, and Cumrun Vafa. 2000. “Knots, Links and Branes at Large N." Journal of High Energy Physics 2000 (11): 007-007. https:// doi.org/10.1088/1126-6708/2000/11/007.

\section{Permanent link}

http://nrs.harvard.edu/urn-3:HUL.InstRepos:41385014

\section{Terms of Use}

This article was downloaded from Harvard University's DASH repository, and is made available under the terms and conditions applicable to Other Posted Material, as set forth at http:// nrs.harvard.edu/urn-3:HUL.InstRepos:dash.current.terms-of-use\#LAA

\section{Share Your Story}

The Harvard community has made this article openly available.

Please share how this access benefits you. Submit a story.

\section{Accessibility}


US-FT-17/00

RUNHETC-2000-38

HUTP-00/A042

hep-th/0010102

\title{
Knots, Links and Branes at Large $N$
}

\author{
J.M.F. Labastida ${ }^{a}$, Marcos Mariño $^{b}$ and Cumrun $\operatorname{Vafa}^{c}$ \\ ${ }^{a}$ Departamento de Física de Partículas, Universidade de Santiago de Compostela \\ E-15706 Santiago de Compostela, Spain \\ ${ }^{b}$ New High Energy Theory Center, Rutgers University \\ Piscataway, NJ 08855, USA \\ ${ }^{c}$ Jefferson Physical Laboratory, Harvard University \\ Cambridge, MA 02138, USA
}

\begin{abstract}
We consider Wilson loop observables for Chern-Simons theory at large $N$ and its topological string dual and extend the previous checks for this duality to the case of links. We find an interesting structure involving representation/spin degeneracy of branes ending on branes which features in the large $N$ dual description of Chern-Simons theory. This leads to a refinement of the integer invariants for links and knots. We illustrate our results with explicit computations on the Chern-Simons side.
\end{abstract}

October 12,2000 


\section{Introduction}

The large $N$ limit of Chern-Simons theory on $\mathbf{S}^{3}$ has been conjectured to be equivalent to topological strings on the blown up conifold geometry [1]. The implications of this conjecture for the Wilson loop observables have been formulated in [2] and checked for the case of the unknot. In particular, this conjecture implies that the knot invariants of Chern-Simons theory can be formulated in terms of new integer invariants [2]. The requisite Wilson loop observables for torus knots were computed in [3] and found to be in agreement with the predictions of this large $N$ conjecture. The large $N$ conjecture has also been checked for some other knots in [4].

The structure of the large $N$ result suggests that arbitrary Wilson loop observables are related on the closed topological string side, to considering the propagation of topological strings in the blown up conifold geometry with a background non-compact D-brane. Thus the results of this duality can be turned around and be used for computations of topological strings with boundaries-a subject which is not very well understood at the present.

The aim of this paper is to extend these results in a number of directions. First we wish to gain a deeper understanding of the structure of the new integer invariants. In particular the results of [3] shows that there are some universal regularities in these numbers that requires explanation. We explain these universal properties in this paper by studying the problem of counting degeneracy of D2 branes ending on D4 branes. Moreover this leads to a refinement of the basic integer invariants introduced in [2]. Secondly we wish to extend these results to links. This involves, on the Chern-Simons computational side, a careful treatment of link invariants consistent with the large $N$ expansion. The results are found to agree with predictions of the large $N$ duality. Thirdly, we construct the relevant Lagrangian D-brane on the topological string side, for a large class of algebraic knots.

The organization of this paper is as follows: In section 2 we review the large $N$ ChernSimons/topological string duality, including the incorporation of Wilson loop observables. In section 3 we analyze the BPS structure of D2 branes ending on D4 branes which leads us to a refinement of the integer invariants associated to knots and links. In section 4 we formulate the link invariants in the context of large $N$ Chern-Simons theory. In section 5 we construct the relevant Lagrangian submanifold associated to a large class of algebraic knots, needed for the closed string dual. Finally, in section 6 we present many explicit results for the refined integer invariants for some knots and links. 


\section{Chern-Simons theory and topological strings}

It was conjectured in [1] that $S U(N)$ Chern-Simons gauge theory on $S^{3}$ at large $N$ is equivalent to topological strings on the non-compact Calabi-Yau threefold, with local geometry given by the $\mathcal{O}(-1)+\mathcal{O}(-1)$ bundle over $\mathbf{P}^{1}$. This conjecture was motivated by considering topological D3-branes for which the worldvolume theory gives rise to $S U(N)$ Chern-Simons theory [5]. Namely one considers the conifold geometry

$$
z_{1}^{2}+z_{2}^{2}+z_{3}^{2}+z_{4}^{2}=\mu
$$

where $z_{i}$ are complex parameters. If we decompose the complex coordinates to real coordinates, i.e., if we write

$$
z_{j}=x_{j}+i p^{j}
$$

then one can see that the above equation has the geometry of $T^{*} \mathbf{S}^{3}$ where $\mathbf{S}^{3}$ is given by $p^{i}=0$, and the $p^{i}$ denote the cotangent directions. If we consider $N$ topological $D 3$ branes wrapped on the $\mathbf{S}^{3}$, then on $\mathbf{S}^{3}$ we get an effective string theory which is an $S U(N)$ Chern-Simons theory [5], at level $k$, where the string coupling is $g_{s}=2 \pi i /(k+N)$ (the shift in the denominator $k \rightarrow k+N$ is the familiar quantum correction in the context of CS theory). The action is

$$
S=\frac{k}{4 \pi} \int_{M} \operatorname{Tr}\left(A \wedge d A+\frac{2}{3} A \wedge A \wedge A\right),
$$

where $A$ is a gauge connection on some $S U(N)$ vector bundle over a three-manifold $M$, which in this case we take to be $\mathbf{S}^{3}$.

The conjecture of [1] is that at large $N$ the D-branes disappear, and instead the conifold undergoes a transition where $\mathbf{S}^{3}$ shrinks to zero size $(\mu \rightarrow 0)$ and a $\mathbf{P}^{1}$ grows whose Kähler parameter $t$ is given by

$$
t=N g_{s}=\frac{2 \pi i N}{k+N}
$$

This conjecture has been checked for the free energy on both sides to all orders in the $1 / N$ expansion. The answer for the partition function on the Chern-Simons side was derived in [6] and on the closed string side the topological string amplitude was computed using M-theory [7] and also using the mathematical definition of topological strings in [8]. This duality has been recently been embedded in the context of type IIA superstrings with $N=1$ supersymmetry in 4 dimensions [9] and it provides a new context where a 
background involving a large number of $D$-branes is equivalent to another background without any $D$-branes.

There is more to the Chern-Simons theory than just the partition function. In particular there is a rich set of observables associated to links in $\mathbf{S}^{3}$ and representations of the gauge group $S U(N)$ [6]. In particular, from the holonomy of the gauge field around a closed loop $\gamma$ in $M$,

$$
U=\mathrm{P} \exp \oint_{\gamma} A
$$

one can construct a natural class of observables, the gauge-invariant Wilson loop operators, which are given by

$$
W_{R}^{\gamma}(A)=\operatorname{Tr}_{R} U
$$

where $R$ denotes an irreducible representation of $S U(N)$. Some of the standard topological invariants that have been considered in the context of Chern-Simons gauge theory are vevs of products of these operators:

$$
\left\langle W_{R_{1}}^{\gamma_{1}} \cdots W_{R_{n}}^{\gamma_{n}}\right\rangle=\frac{1}{Z(M)} \int[\mathcal{D} A]\left(\prod_{i=1}^{n} W_{R_{i}}^{\gamma_{i}}\right) \mathrm{e}^{i S},
$$

where $Z(M)$ is the partition function of the theory. These vevs are functions of the variables

$$
q=\mathrm{e}^{g_{s}}=\exp \left[\frac{2 \pi i}{k+N}\right], \quad \lambda^{-1}=q^{-N}=\mathrm{e}^{-t} .
$$

In the context of topological strings, i.e., with $N$ D3 branes on $\mathbf{S}^{3}$ embedded in the conifold background, it is natural to ask how these observables can arise. This was answered in 22]: Consider a number of $\gamma_{\alpha} \in \mathbf{S}^{3}, \alpha=1, \cdots, L$. Then to each one of them one associates a Lagrangian submanifold $D_{\alpha}$ which intersects $\mathbf{S}^{3}$ on $\gamma_{\alpha}$. This is obtained by appending to each point of $\gamma_{\alpha}$ a two dimensional subspace $\mathbf{R}^{2}$ which is the set of momentum vectors $p$ orthogonal to the velocity vector $d \gamma_{\alpha} / d s$. Note that the topology of each $D_{\alpha}$ is $\mathbf{R}^{2} \times \mathbf{S}^{1}$. We consider placing $M_{\alpha}$ branes on the cycle $D_{\alpha}$. Since these are non-compact we can consider them as non-dynamical. In particular on each of them lives an $S U\left(M_{\alpha}\right)$ gauge fields which we consider as classical. Let $V_{\alpha}$ denote the holonomy of this gauge field along the $\gamma_{\alpha} \in D_{\alpha}$. As far as the Chern-Simons gauge theory on $\mathbf{S}^{3}$ is concerned we get additional massless fields living on the $\gamma_{\alpha}$ corresponding to open strings stretched between branes on $D_{\alpha}$ and $\mathbf{S}^{3}$. Integrating them out leads (as discussed in [2]) to the operator

$$
Z\left(U_{\alpha}, V_{\alpha}\right)=\exp \left[\sum_{\alpha=1}^{L} \sum_{n=1}^{\infty} \frac{1}{n} \operatorname{Tr} U_{\alpha}^{n} \operatorname{Tr} V_{\alpha}^{n}\right]
$$


In this operator the trace is taken over the fundamental representation 1 .

If one can evaluate the expectation value $\left\langle Z\left(U_{\alpha}, V_{\alpha}\right)\right\rangle=\exp \left(F\left(V_{\alpha}\right)\right)$ for all links, then effectively one can compute all the topological observables of Chern-Simons theory (2.4) by rewriting the holonomy in representation $R, \operatorname{Tr}_{R} U$ in terms of the trace of powers of holonomy in fundamental representation, appearing in $Z\left(U_{\alpha}, V_{\alpha}\right)$.

It is natural then to ask how these correlators behave at large $N$. Since according to the conjecture [1] we should be effectively ending up with topological strings on the blown up conifold geometry where the D-branes wrapped around $\mathbf{S}^{3}$ have disappeared, we need to know what happens to the other D-branes wrapped over the non-compact $D_{\alpha}$ cycles after this transition. Geometrically it is clear that they cannot disappear (as the only thing that shrinks is the $\mathbf{S}^{3}$ ) and so they should manifest themselves as some Lagrangian submanifolds on the other side. Let us continue to denote the corresponding Lagrangian submanifold by $D_{\alpha}$. Moreover, there should be a distinguished $\mathbf{S}^{1}$ on the Lagrangian submanifold after the transition, for which we consider the holonomy $V_{\alpha}$. Thus the statement of the conjecture would be that $F\left(V_{\alpha}\right)$ can be computed by considering the topological strings on the blown up geometry with extra D-branes $D_{\alpha}$. In particular

$$
F\left(V_{\alpha}\right)=\sum_{g=0}^{\infty} \sum_{h=1}^{\infty} \sum_{\alpha, n_{1}, \cdots, n_{h}} g_{s}^{2 g-2+h} F_{g ; n_{1}, \cdots, n_{h}}^{\alpha} \operatorname{Tr} V_{\alpha_{1}}^{n_{1}} \cdots \operatorname{Tr} V_{\alpha_{h}}^{n_{h}}
$$

where $F_{g ; n_{1}, \cdots, n_{h}}^{\alpha}$ denotes the topological string amplitude on the blown up geometry with genus $g$ and with $h$ holes, where the $i$-th hole is mapped to the $D_{\alpha_{i}}$ brane and winds around the corresponding circle $n_{i}$ times. The computation of these amplitudes is very difficult, and no techniques are currently available to do a direct computation. However, some general features of topological amplitudes can be deduced. This was done in [2] following the idea of [7] [10], which relates the degeneracy of D2 branes, including both their charge (wrapping around 2-cycles) and spin, to the topological string amplitudes. The novel feature in the case at hand is that one has to consider branes ending on the Lagrangian submanifold. Moreover, in addition to the "bulk" charge and spin, the D2 brane also forms a representation of $S U(M)$. It was shown in [2] that by relating the topological string amplitudes to type IIA superstring amplitudes in 2 dimensions with background D4

1 In what follows, when no representation is indicated in a trace, it should be understood that it must be taken in the fundamental representation. 
branes, and considering the contribution of D2 branes ending on D4 branes to topological string amplitudes, one can deduce the following structure for the partition function:

$$
F(V)=\sum_{d=1}^{\infty} \sum_{R} f_{R}\left(q^{d}, \lambda^{d}\right) \operatorname{Tr}_{R} \frac{V^{d}}{d}
$$

where

$$
f_{R}(q, \lambda)=\sum_{s, Q} \frac{N_{R, Q, s}}{q^{\frac{1}{2}}-q^{-\frac{1}{2}}} \lambda^{Q} q^{s} .
$$

Here, the $N_{R, Q, s}$ are integers and denote the degeneracy of D2 branes of bulk charge $Q$ with 2 d spacetime spin $s$ and $S U(M)$ representation $R$. The $Q$, for a given representation $R$, denotes an element of the relative homology $H_{2}(X, D)$ where $X$ is the CY $\mathcal{O}(-1)+\mathcal{O}(-1)$ over $\mathbf{P}^{1}$ and $D$ is the Lagrangian submanifold. The difference of two $Q$ 's is an integer for any fixed $R$. In the examples we will deal with it turns out that $Q$ 's are integer or half-integer. Here we have written the partition function for the case of a single D-brane, but that can be easily generalized. The integrality properties of $N_{R, Q, s}$ are rather nontrivial and this gives a strong constraint on any proposed answer for the topological string amplitudes involving D-branes.

To check the large $N$ conjecture for the Wilson loop observables one has to overcome a number of obstacles. On the gauge theory side we have to compute the Chern-Simons observables for arbitrary links and representations. On the gravity side (i.e., after the transition) we need to construct the $D_{\alpha}$ 's. Finally we have to find a way to compute the topological string amplitudes in the presence of these D-branes, which is equivalent to computing the degeneracy numbers $N_{R, Q, s}$. 2

These questions were answered in [2] for a very simple observable, namely the unknot, which is just a single $\gamma$ which is not knotted. The Lagrangian submanifold after the transition side was identified for this case (by identifying it as the invariant locus of an anti-holomorphic involution). In particular it intersected the $\mathbf{P}^{1}$ on an equator. It was shown in that case that the geometry of D2 branes is particularly simple and there are only two D2 branes ending on the Lagrangian submanifold: one corresponding to the D2 brane wrapped over the northern hemisphere, and the other over the southern hemisphere.

2 By analytic continuation one can also express the topological string amplitudes in terms of the conjugate quantum numbers by flipping the sign, i.e., $N_{R^{*},-Q,-s}=-N_{R, Q, s}$, where $R^{*}$ denotes the conjugate representation. 
These have $Q$ charge $\pm 1 / 2$ and $\operatorname{spin} s=0$, and are in the fundamental representation, namely $N_{\square, \pm 1 / 2,0}= \pm 1$ and the rest of the $N_{R, Q, s}$ are zero.

To further test the above predictions at large $N$, more interesting knots were considered in [3]. In particular a class of knots known as $(n, m)$ torus knots were studied. Already the Chern-Simons computation is very non-trivial for these knots, as one has to find a way to compute arbitrary number of product of the trace of the holonomy observable in all representations of $S U(N)$. Moreover one has to relate this with the particular combinations of observables given in (2.8)(2.9). For this comparison it turns out natural to reexpress the Chern-Simons observables in terms of powers of $U$ in the fundamental representation. In particular if $R$ is a representation of $S U(N)$ with $\ell$ boxes then $\operatorname{Tr}_{R} U$ is a universal polynomial in $\operatorname{Tr} U^{k}$ in the fundamental representation of total degree $\ell$. Then the question of computation gets transformed to the computation of product of various powers of $\operatorname{Tr} U^{k}$. At the end of the day, what one finds is that if one knows the expectation value of the single $\left\langle\operatorname{Tr}_{R} U\right\rangle$ for any representation $R$ of $S U(N)$ with up to $\ell$ boxes, one can find $f_{R^{\prime}}$ for representation $R^{\prime}$ of $S U(M)$ with up to $\ell$ boxes. This gives a recursive way to compute the $f_{R^{\prime}}$ organized in increasing order in terms of the number of boxes. Let us review this in a little more detail.

As shown in [3], to compare the large $N$ topological string predictions (2.8) (2.9) with the Chern-Simons computation, it is convenient to introduce the following basis on the space of Wilson loop operators:

$$
\Upsilon_{\vec{k}}(U)=\prod_{j=1}^{\infty}\left(\operatorname{Tr} U^{j}\right)^{k_{j}},
$$

which are labeled by a vector $\vec{k}$ of nonnegative integers, and $U$ is the holonomy (2.2). Given such a vector, we define:

$$
\ell=\sum j k_{j}, \quad|\vec{k}|=\sum k_{j} .
$$

We can associate to any vector $\vec{k}$ a conjugacy class $C(\vec{k})$ of the permutation group $S_{\ell}$. This class has $k_{1}$ cycles of length $1, k_{2}$ cycles of length 2 , and so on. The number of elements of the permutation group in such a class is given by [11]

$$
|C(\vec{k})|=\frac{\ell !}{\prod k_{j} ! \prod j^{k_{j}}} .
$$


Notice that the vectors $\vec{k}$ with $\sum_{j} j k_{j}=\ell$ are in one-to-one correspondence with the partitions of $\ell$. We also define generalized connected vevs as follows: first, associate to any $\vec{k}$ the polynomial $p_{\vec{k}}(x)=\prod_{j} x_{j}^{k_{j}}$ in the variables $x_{1}, x_{2}, \ldots$ We then define the "connected" coefficients $a_{\vec{k}}^{(c)}$ :

$$
\log \left(1+\sum_{\vec{k}} \frac{|C(\vec{k})|}{\ell !} a_{\vec{k}} p_{\vec{k}}(x)\right)=\sum_{\vec{k}} \frac{|C(\vec{k})|}{\ell !} a_{\vec{k}}^{(c)} p_{\vec{k}}(x)
$$

We also introduce the following notation for the vevs of the operators in (2.10):

$$
G_{\vec{k}}=\left\langle\Upsilon_{\vec{k}}(U)\right\rangle
$$

Using (2.8) (2.9) one deduces the following relation [3]:

$$
G_{\vec{k}}^{(c)}(U)=\sum_{n \mid \vec{k}} n^{|\vec{k}|-1} \sum_{R} \chi_{R}\left(C\left(\vec{k}_{1 / n}\right)\right) f_{R}\left(q^{n}, \lambda^{n}\right),
$$

where $\chi_{R}(C(\vec{k}))$ denotes the character of the representation $R$ of the symmetric group for the conjugacy class $C(\vec{k})$ specified by the vector $\vec{k}$. In this equation, the vector $\vec{k}_{1 / n}$ is defined as follows. Fix a vector $\vec{k}$, and consider all the positive integers that satisfy the following condition: $n \mid j$ for every $j$ with $k_{j} \neq 0$. When this happens, we will say that " $n$ divides $\vec{k}$," and we will denote this as $n \mid \vec{k}$. We can then define the vector $\vec{k}_{1 / n}$ whose components are:

$$
\left(\vec{k}_{1 / n}\right)_{i}=k_{n i}
$$

The vectors which satisfy the above condition and are "divisible by $n$ " have the structure $\left(0, \ldots, k_{n}, 0, \ldots, 0, k_{2 n}, \ldots\right)$, and the vector $\vec{k}_{1 / n}$ is then given by $\left(k_{n}, k_{2 n}, \ldots\right)$.

One can extract the generating functions $f_{R}(q, \lambda)$ from the connected vevs in ChernSimons theory, using the relation (2.15). This was done in [3] to check, for the torus knots, the structure predicted in [2] for the large $N$ behavior of the Wilson loop observables. In particular the integrality properties of the topological string amplitudes were verified. The results of [3] strongly suggest that for non-trivial torus knots, the integer invariants $N_{R, Q, s}$ are non-vanishing for all representations. Moreover for each representation $R$, there are a finite number of values of $Q, s$ for which the $N$ 's are non-vanishing. These $N_{R, Q, s}$ would give the number of D2 branes in the blown up geometry which end on the Dbranes wrapping around the Lagrangian submanifold corresponding to torus knots after the transition. These Lagrangian submanifolds were not constructed in [3], but we will present 
a proposal for them in section 5 of this paper. Even knowing the Lagrangian submanifold is not sufficient to give the integer invariants, since there are no known techniques in complicated enough cases as we are encountering to directly compute $N_{R, Q, s}$ 's. So this large $N$ computation of Chern-Simons knot invariants should be viewed as a powerful technique for computing degeneracies of branes in this geometry.

Even though all the predicted aspect of the large $N$ theory were checked for torus knots in [3], more structure was found in [3] which needed further explanation. In particular it was noted there that the topological string answer predicts certain surprising universal relations among the BPS degeneracies. For example a simple (inductive) reformulation of the results in [3] shows that

$$
N_{R, Q, s}=(-1)^{\ell-1} N_{R^{t}, Q,-s}
$$

where $R^{t}$ denotes the $S U(M)$ representation which has the transposed Young tableau of the one corresponding to $R$, and $\ell$ is the number of boxes in the Young tableau. In fact this follows by checking the agreement of the large $N$ expansion encoded in terms of the topological closed string amplitude (2.7) with that given in (2.8) (2.9). To see this notice that, after performing an analytic continuation to a series involving only positive $n_{i},(2.7)$ can be written as

$$
F(V)=\sum_{\vec{k}} \sum_{g=0}^{\infty} F_{g, \vec{k}}(\lambda) g_{s}^{2 g-2+|\vec{k}|} \Upsilon_{\vec{k}}(V)
$$

where $k_{i}$ is the number of $i$ 's in the $h$-uple $\left(n_{1}, \cdots, n_{h}\right)$, so that $|\vec{k}|=h$. In the context of Chern-Simons theory, (2.18) is nothing but the usual $1 / N$ expansion of the connected vevs of Wilson loops. Note that the parity of the power of $g_{s}$ in (2.18) correlates with the number of holes, and translating this to (2.8)(2.9) using Frobenius relation and

$$
\chi_{R^{t}}(C(\vec{k}))=(-1)^{|\vec{k}|+\ell} \chi_{R}(C(\vec{k})),
$$

yields the above relation among $N_{R, Q, s}$ 's. There were also additional vanishing relations noted in [3] from comparing (2.18) and (2.8)(2.9) which were also in need of explanation. In this paper we will be able to explain all those relations (using some plausibility assumptions) by studying more carefully the degeneracy of D2 branes ending on D4 branes and in particular we will have a reformulation of $N_{R, Q, s}$ in terms of other more basic integer quantities $\widehat{N}_{R, Q, g}$ which are not restricted.

Further checks for the large $N$ predictions of Wilson loop observable, involving certain knots up to nine crossings, were recently done in [4] using the techniques of [12]. 


\section{Degeneracy of D2-branes ending on D4-branes}

As discussed before, there are hints that the numbers $N_{R, Q, s}$ satisfy certain universal relations. We wish to derive this fact from the viewpoint of counting the degeneracy of D2 branes ending on D4 branes in the stringy realization of topological strings with D-branes.

Consider type IIA strings on a Calabi-Yau threefold (which for most of the applications we have in mind will be non-compact). Suppose we have $M$ D4-branes wrapping a 3-dimensional Lagrangian submanifold of the Calabi-Yau, and filling a 2-dimensional spacetime subspace of $\mathbf{R}^{4}$. Suppose also that for the Lagrangian submanifold we have $b_{1}=1$. As discussed in [2] this gives rise, in the $\mathbf{R}^{2}$ subspace, to a $U(1)^{M}$ magnetic gauge theory. We will be interested in the degeneracy of D2 branes ending on D4 branes in this geometry. These can be viewed as having some $U(1)^{M}$ charge. By the $S_{M}$ permutation invariance of the D4 branes, it follows that the particles will form representations of $U(1)^{M} / S_{M}$, which can also be viewed as linear combination of representations of $U(M)$. Let us label such a representation with $R$. The D2-brane will also have some charge $Q$ corresponding to which 2-cycle (in the sense of relative homology) of the Calabi-Yau it wraps around. It also has some $S O(2)$ spin $S$. To define this more precisely, we have to consider the M-theory description of this geometry [2]. This means that we consider M-theory on CY 3-fold with M5 branes filling $\mathbf{R}^{3}$ and wrapping over the Lagrangian submanifold in the CY. We relate the $S O(2)$ spin $S$ to the 3-dimensional rotation symmetry on the non-compact worldvolume of the M5 brane. There is also an $S O(2)$ R-symmetry for 4 supercharges in $d=3$. For BPS states a combination of supercharges which is neutral under $S_{L}=S+R$ annihilates the state, while the other supercharge which changes $S_{L}$, but is neutral under $S_{R}=S-R$, generates the BPS multiplet. This is very similar to the BPS structure for a theory in 5 dimensions with 8 supercharges (such as CY 3-fold compactifications of M-theory). In that case one has the rotation group $S O(4)=S U(2)_{L} \times S U(2)_{R}$ and the active supercharge of the BPS states resides in one of the $S U(2)$ 's. This was in fact strongly used in relating the degeneracy of D2 branes wrapped around cycles of CY 3-fold with the topological string amplitudes [10]. The $S_{L}$ and $S_{R}$ of the $3 \mathrm{~d}$ theory we have been discussing correspond to the $J_{L}^{3}$ and $J_{R}^{3}$ of these $S U(2)$ 's respectively. In fact the presence of the M5 brane breaks the $S O(4)$ rotation symmetry of 5 d theory to $S O(2)_{S} \times S O(2)_{R}$ which gives us the above identification of $S_{L, R}$ with $J_{L, R}^{3}$.

The number of BPS states may change in general, if two short multiplets combine to a bigger multiplet. There is a way to define an index which is the net number of BPS states 
which cannot recombine. Namely, if we take a trace over all BPS states of a given charge and representation quantum numbers, as well as a fixed $S_{L}$, weighted with $\operatorname{Tr}(-1)^{2 S_{R}}$, one gets zero for pairs of short multiplets that can combine to a long multiplet. Thus this combination is invariant under deformation. The number $N_{R, Q, s}$ refers to this net number where $s=S_{L}$.

We are now ready to study the net degeneracy of D2-branes ending on D4-branes, in the sense defined above. Similar to what was done in the context of the closed string case [10] we first consider the situation where we have a D2-brane of a fixed genus $g$ with $\ell$ holes ending on the D4-brane. We will assume for simplicity that if there are moduli for these D2-branes the genus and the number of holes does not change over all of this moduli space. Let us call this moduli space $\mathcal{M}_{g, \ell}$. We will take this to be the moduli space with $\ell$ ordered holes (later we will divide by the action of permutation group $S_{\ell}$ to find the physical number of states).

As discussed in [10] we have to study the moduli space to D2-branes ending on D4branes together with a flat bundle on it. For a genus $g$ surface with $\ell$ holes this gives rise to the Jacobian $J_{g, \ell}=\mathbf{T}^{2 g+\ell-1}$. The assumption that there is no degeneration of the Riemann surface along the moduli, means that the Jacobian is always a constant $\mathbf{T}^{2 g+\ell-1}$ (otherwise we would have complications similar to the ones discussed in 10] in the context of closed strings). There is only one more ingredient: for each boundary we should decide which of the $M$ branes it lives on. Thus we naturally get, for the Hilbert space, a tensor product of $F^{\otimes \ell}$, where $F$ is the fundamental representation of $S U(M)$. Therefore, the Hilbert space associated naturally to the above geometrical configuration is given by

$$
F^{\otimes \ell} \otimes H^{*}\left(J_{g, \ell}\right) \otimes H^{*}\left(\mathcal{M}_{g, \ell}\right)
$$

An important point is that this Hilbert space is associated with the moduli space of $\ell$ distinguished holes, which is not physical, and we have to mod out by the action of the permutation group $S_{\ell}$. We can factor out the cohomology of the Jacobian $\mathbf{T}^{2 g}$ of the "bulk" Riemann surface, $H^{*}\left(\mathbf{T}^{2 g}\right)$, since the permutation group does not act on it. The projection onto the symmetric piece can be easily done using the Clebsch-Gordon coefficients $C_{R} R^{\prime}{ }^{\prime \prime}$ of the permutation group $S_{\ell}$ :

$$
\begin{aligned}
& \operatorname{Sym}\left(F^{\otimes \ell} \otimes H^{*}\left(\left(\mathbf{S}^{1}\right)^{\ell-1}\right) \otimes H^{*}\left(\mathcal{M}_{g, \ell}\right)\right)= \\
& \sum_{R R^{\prime} R^{\prime \prime}} C_{R R^{\prime} R^{\prime \prime}} \mathbf{S}_{R}\left(F^{\otimes \ell}\right) \otimes \mathbf{S}_{R^{\prime}}\left(H^{*}\left(\left(\mathbf{S}^{1}\right)^{\ell-1}\right)\right) \otimes \mathbf{S}_{R^{\prime \prime}}\left(H^{*}\left(\mathcal{M}_{g, \ell}\right)\right),
\end{aligned}
$$


where the subscripts of the representation of the vector spaces mean projecting the space to the corresponding subspace. The space $\mathbf{S}_{R}\left(F^{\otimes \ell}\right)$ is nothing but the vector space underlying the irreducible representation $R$ of $S U(M)$. In other words, even though to begin with $R$ labels representations of $S_{\ell}$ the projection also leads to a definite representation of the $S U(M)$ group, due to the relation between representation of symmetric groups and that of $S U(M)$. So in this sense the same Young tableau will denote also the representation content of $S U(M)$. We will thus use the label $R$ in both senses.

We have to be more specific about the action of the permutation group on the cohomology elements. Let's denote by $H=H^{*}\left(\mathbf{S}^{1}\right)$ the cohomology of the circle. Although the permutation group $S_{\ell}$ acts in a natural way on a Riemann surface with $\ell$ boundaries, there are only $\ell-1$ independent one-forms associated to the boundary. This is because the one-forms $d \theta_{i}, i=1, \cdots, \ell$, which are Poincaré dual to the holes in the Riemann surface, satisfy $\sum_{i} d \theta_{i}=0$. The procedure to construct the Hilbert space $\mathbf{S}_{R}\left(H^{\ell-1}\right)$ is then as follows. We consider the Hilbert space $H^{\ell}$ generated by $\ell$ fermion fields $\psi_{i}, i=1, \cdots, \ell$ acting on the vacuum $|0\rangle$ and we decompose it with respect to the different representations $R$ by using the Young symmetrizers of the corresponding tableaux, and taking into account the Grassmann nature of the fermions. Finally, we impose the linear constraint $\sum_{i} \psi_{i}=0$. It is instructive to consider the simple case $\ell=2$ in some detail. This would for example arise for the D2-brane with the topology of annulus. The space $H^{2}$ is spanned by the four states $|0\rangle, \psi_{1,2}|0\rangle$ and $\psi_{1} \psi_{2}|0\rangle$. The relevant permutation group $S_{2}$ corresponds to permuting $\psi_{1} \leftrightarrow \psi_{2}$. Projecting onto the symmetric and antisymmetric subspaces, we find:

$$
\text { ш : }|0\rangle,\left(\psi_{1}+\psi_{2}\right)|0\rangle \quad ; \quad \text { 日: }\left(\psi_{1}-\psi_{2}\right)|0\rangle, \quad \psi_{1} \psi_{2}|0\rangle \text {. }
$$

Using that $\psi_{1}+\psi_{2}=0$, the spectrum turns out to be:

$$
\text { 口: }|0\rangle, \quad \text { 曰: } \psi_{1}|0\rangle .
$$

To assign spins to these states, first we note from the discussion above that the relevant notion of spin is $S_{L}$, and with respect to $S_{L}$ the fermions associated to the choice of the flat connection on Riemann surface carry spin $1 / 2$. In other words, $\psi_{i}$ have spin $1 / 2$. However this does not fix the spin assignment of all the states, as we have to choose a spin for the ground state. It is natural to choose the spin for the ground state in such a way that in a given multiplet the average spin is zero. This is what we will assume (and is consistent with the choice which naturally arises from the duality with knots in Chern-Simons theory). In 
the case at hand the two states differ in spin by $1 / 2$, and so the symmetric choice of spin assignment is spin $\mp 1 / 4$. With our choice of normalization we call this $s= \pm \frac{1}{2}$. The same procedure gives for $\ell=3$ :

$$
\text { 口 : }|0\rangle, \quad \text { 口: }\left(\psi_{1}+\psi_{2}\right)|0\rangle, \quad \text { 目: } \psi_{1} \psi_{2}|0\rangle,
$$

with spin assignments $-1 / 2,0,1 / 2$ i.e., $s=-1,0,1$.

It is clear how to proceed to find the spin content of various representation that arise in this way, when we have more holes. The symmetric spectrum, corresponding to a Young tableau with $\ell$ boxes and only one row, is given by the vacuum $|0\rangle$, and we assign it spin $-(\ell-1) / 2$. Let's now consider the states that are obtained acting with one fermion $\psi_{i}$ on the vacuum. There are $\ell$ of them, forming a reducible representation $(\ell)$ of $S_{\ell}$ which decomposes in reducible representations as follows:

$$
(\ell)=(\ell-1) \oplus(1)
$$

The first summand corresponds to the standard representation $V$ of $S_{\ell}$, with a Young tableau of the form:

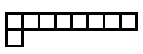

with $\ell-1$ boxes in the first row. The second summand corresponds to the trivial representation generated by $\left(\sum_{i} \psi_{i}\right)|0\rangle$, which we are putting to zero. To generate the rest of the spectrum, we have just to take the antisymmetrized tensor products $\wedge^{d} V$ (since $V$ is fermionic). These are irreducible representations of $S_{\ell}$ and are called hook representations, since their Young tableau is of the form

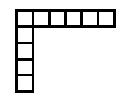

with $\ell-d$ boxes in the first row. We have then obtained the spin/representation content of the spectrum: the Hilbert spaces $\mathbf{S}_{R}\left(H^{\ell-1}\right)$ are nonempty only for hook representations of the form (3.8), and in this case they contain one state of statistics $(-1)^{d}$ and total spin $-(\ell-1) / 2+d$, which is equal to the spin of the vacuum plus $d$ units of the $d$ fermionic fields that appear in $\wedge^{d} V$.

It is useful to encode the spectrum associated to a Hilbert space $\mathcal{H}$ in a generating function $\operatorname{Tr}_{\mathcal{H}}(-1)^{F} q^{s}$. The degeneracy and the spin content of the contribution of a 
Riemann surface with $\ell$ boundaries in representation $R$ is summarized in the generating function:

$$
S_{R}(q)=\operatorname{Tr}_{\mathbf{S}_{R}\left(H^{\ell-1}\right)}(-1)^{F} q^{s} .
$$

In fact that is precisely the contribution of the boundary states to $f_{R}$. As we have argued, the generating function corresponding to the hook representation $R$ is given by,

$$
S_{R}(q)=(-1)^{d} q^{-\frac{\ell-1}{2}+d},
$$

and $S_{R}(q)=0$ for the rest of the representations. For example for the case of two holes one has that $S_{\square}(q)=q^{-1 / 2}$ and $S_{日}(q)=-q^{1 / 2}$, while for $\ell=3$ one has

$$
S_{\text {口四 }}(q)=q^{-1}, \quad S_{\text {甲 }}(q)=-1, \quad S_{\text {日 }}(q)=q ，
$$

in agreement with (3.4) and (3.5).

The generating functions $S_{R}(q)$ have two properties that will be needed later. First of all, they satisfy

$$
S_{R}\left(q^{-1}\right)=(-1)^{\ell-1} S_{R^{t}}(q),
$$

which is a direct consequence of the explicit expression (3.10). The second property that they have is the following. Define the following polynomials, which are labeled by a conjugacy class $C(\vec{k})$ of $S_{\ell}$ :

$$
P_{\vec{k}}(q)=\sum_{R} \chi_{R}(C(\vec{k})) S_{R}(q)
$$

which can be understood as the graded trace of the element $C(\vec{k})$ on the total Hilbert space $H^{\ell-1}$ :

$$
\operatorname{Tr}_{H^{\ell-1}}(-1)^{F} C(\vec{k}) q^{s}
$$

If $C(\vec{k})$ is the trivial permutation, we just get the generating function for $H^{\ell-1}$ :

$$
q^{-\frac{\ell-1}{2}}(1-q)^{\ell-1}=\frac{\left(q^{-\frac{1}{2}}-q^{\frac{1}{2}}\right)^{\ell}}{q^{-\frac{1}{2}}-q^{\frac{1}{2}}} .
$$

For nontrivial conjugacy classes, with $k_{1}$ one-cycles, $k_{2} 2$-cycles, and so on, the above trace can be computed on $H^{\ell}$ by grouping the fermions as indicated by the conjugacy class:

$$
\psi_{\mu_{1}} \cdots \psi_{\mu_{k_{1}}} \psi_{\mu_{1} \nu_{1}} \cdots \psi_{\mu_{k_{2}} \nu_{k_{2}}} \cdots
$$


where $\psi_{\mu_{i} \nu_{i}} \ldots=\psi_{\mu_{i}} \psi_{\nu_{i}} \cdots$ are "composites" which for a cycle of length $j$ have spin $j$, and there are $k_{j}$ of them. It is then easy to show that

$$
P_{\vec{k}}(q)=\frac{\prod_{j}\left(q^{-\frac{j}{2}}-q^{\frac{j}{2}}\right)^{k_{j}}}{q^{-\frac{1}{2}}-q^{\frac{1}{2}}}
$$

where we have factored out a $q^{-\frac{1}{2}}-q^{\frac{1}{2}}$ which comes from imposing the constraint $\sum_{i} \psi_{i}=$ 0. This expression will be useful later on.

We can now give the expression of $f_{R}(q, \lambda)$ in terms of the structure we have analyzed. Note that since the cohomology of $\mathcal{M}_{g, \ell}$ is represented by fermions which only carry "right" spin $S_{R}=S-R$ [10] we do not need to know their actual cohomology degree in connection with the topological amplitudes which are only sensitive to $S_{L}=S+R$. Define the integers

$$
\widehat{N}_{R, g, Q}=\chi\left(\mathbf{S}_{R}\left(H^{*}\left(\mathcal{M}_{g, \ell}\right)\right)\right)
$$

Then collecting the results from our discussion above, we have

$$
f_{R}(q, \lambda)=\sum_{g \geq 0} \sum_{Q} \sum_{R^{\prime}, R^{\prime \prime}} C_{R R^{\prime} R^{\prime \prime}} S_{R^{\prime}}(q) \widehat{N}_{R^{\prime \prime}, g, Q}\left(q^{-\frac{1}{2}}-q^{\frac{1}{2}}\right)^{2 g-1} \lambda^{Q},
$$

where $\left(q^{-\frac{1}{2}}-q^{\frac{1}{2}}\right)^{2 g}$ comes from the bulk of the Riemann surface, as in [10], and the extra $\left(q^{-\frac{1}{2}}-q^{\frac{1}{2}}\right)^{-1}$ comes from the Schwinger computation [2]. It is useful to introduce the generating functions:

$$
\widehat{f}_{R}(q, \lambda)=\sum_{g \geq 0} \sum_{Q} \widehat{N}_{R, g, Q}\left(q^{-\frac{1}{2}}-q^{\frac{1}{2}}\right)^{2 g-1} \lambda^{Q}
$$

We then have the relation:

$$
f_{R}(q, \lambda)=\sum_{R^{\prime}} M_{R R^{\prime}}(q) \widehat{f}_{R}(q, \lambda)
$$

where the matrix $M_{R R^{\prime}}(q)$ is given by

$$
M_{R R^{\prime}}(q)=\sum_{R^{\prime \prime}} C_{R R^{\prime} R^{\prime \prime}} S_{R^{\prime \prime}}(q)=\sum_{\vec{k}} \frac{|C(\vec{k})|}{\ell !} \chi_{R}(C(\vec{k})) \chi_{R^{\prime}}(C(\vec{k})) P_{\vec{k}}(q) .
$$

To obtain this expression, we have taken into account that the Clebsch-Gordon coefficients are given by:

$$
C_{R R^{\prime} R^{\prime \prime}}=\sum_{\vec{k}} \frac{|C(\vec{k})|}{\ell !} \chi_{R}(C(\vec{k})) \chi_{R^{\prime}}(C(\vec{k})) \chi_{R^{\prime \prime}}(C(\vec{k})) .
$$


The matrix (3.22) is invertible, and it is easy to show that the inverse is given by the last line of (3.22) but after substituting $P_{\vec{k}}(q)$ by $1 /\left(P_{\vec{k}}(q)\right)$. This means that, once we have computed all the $f_{R}$ for a fixed $\ell$, we can obtain the new generating functions $\widehat{f}_{R}(q, \lambda)$ using the explicit expression for $M_{R R^{\prime}}^{-1}(q)$. Clearly, from the Chern-Simons point of view it is highly nontrivial that the polynomials obtained in this way have the structure (3.20).

So far we have analyzed the representation and spin content arising from a D2 brane whose moduli can change in a Calabi-Yau manifold, but we have assumed that its topology (the genus $g$ and the number of holes) does not change. This is not the most general situation. However as was shown in the closed string case [10], by a physical reasoning in target space, studying D2 branes as if they have a fixed genus gives a structure for the topological string answer that is in fact the generic case. This has also been understood more directly by studying the degeneration structure of Riemann surfaces in the moduli space of D2 brane [13]. It is plausible to assume that the same holds true for the open string case and that the expression we have found for $f_{R}(q, \lambda)$ in terms of integers $\widehat{N}_{R, g, Q}$ is more generally valid. It would be interesting to verify this. Note, for example, that when we write $g$ in $\widehat{N}_{R, g, Q}$ we do not strictly mean a genus $g$ surface. More precisely this can arise from a D2 brane with genus bigger than or equal to $g$, which at some points along the moduli degenerates to a genus $g$ surface, as in [13]. Also the number of holes on the Riemann surface is greater than or equal to the number of boxes in $R$.

On the physics side we will have to develop what these integers directly compute. In particular the notion of the "bulk spin" $g$ must have an intrinsic physical meaning in the target space description. Moreover one should be able to understand directly from target space reasoning how the $\mathbf{S}_{R}\left(H^{\ell-1}\right)$ arises and how the Clebsch-Gordon coefficients $C_{R R^{\prime} R^{\prime \prime}}$ arise. At any rate we will find further evidence, from the computations of link invariants of Chern-Simons theory that this picture is correct.

One important advantage of the formulation (3.19) is that the relation (2.17) is now manifest. From (3.23), it follows that $C_{R R^{\prime t} R^{\prime \prime t}}=C_{R R^{\prime} R^{\prime \prime}}$, and using (3.12), one easily derives (2.17). Notice that, in contrast to the integers $N_{R, Q, s}$, the new integers $\widehat{N}_{R, g, Q}$ are all independent. This shows that, although the generating functions (3.20) and (3.19) are equivalent (since they are related by an invertible matrix), the invariants introduced in (3.18) are more fundamental than the integers appearing in (2.9).

Before exploring further consequences of the structure result (3.19), let's write it in some detail for representations with $\ell=1,2,3$. For $\ell=1$, we have:

$$
f_{\square}(q, \lambda)=\sum_{Q} \sum_{g \geq 0} \widehat{N}_{R, g, Q}\left(q^{-\frac{1}{2}}-q^{\frac{1}{2}}\right)^{2 g-1} \lambda^{Q},
$$


where we have taken into account that $S_{\square}(q)=1$. Since $f_{\square}(\lambda, q)$ is just the unnormalized HOMFLY polynomial of the knot [3], the above result is in perfect agreement with the fact that the HOMFLY polynomial can be written in terms of even powers of the variable $q^{-\frac{1}{2}}-q^{\frac{1}{2}}[14]$ [15. For $\ell=2$, we have

$$
\begin{aligned}
f_{\square}(q, \lambda) & =\sum_{Q} \sum_{g \geq 0}\left(q^{-\frac{1}{2}} \widehat{N}_{\square, g, Q}-q^{\frac{1}{2}} \widehat{N}_{\text {日, }, g, Q}\right)\left(q^{-\frac{1}{2}}-q^{\frac{1}{2}}\right)^{2 g-1} \lambda^{Q}, \\
f_{\text {日 }}(q, \lambda) & =\sum_{Q} \sum_{g \geq 0}\left(-q^{\frac{1}{2}} \widehat{N}_{\square, g, Q}+q^{-\frac{1}{2}} \widehat{N}_{\text {日, }, Q, Q}\right)\left(q^{-\frac{1}{2}}-q^{\frac{1}{2}}\right)^{2 g-1} \lambda^{Q} .
\end{aligned}
$$

Finally, for $\ell=3$, we find:

$$
\begin{aligned}
& f_{\text {口四 }}(q, \lambda)=\sum_{Q} \sum_{g \geq 0}\left(q^{-1} \widehat{N}_{\text {Ш }, g, Q}-\widehat{N}_{\text {甲, }, Q, Q}+q \widehat{N}_{\text {日, }, Q, Q}\right)\left(q^{-\frac{1}{2}}-q^{\frac{1}{2}}\right)^{2 g-1} \lambda^{Q}, \\
& f_{\boxminus}(q, \lambda)=\sum_{Q} \sum_{g \geq 0}\left(-\widehat{N}_{\text {Ш }, g, Q}+\left(q+q^{-1}-1\right) \widehat{N}_{\text {甲, }, g, Q}-\widehat{N}_{\text {日, }, Q}\right)\left(q^{-\frac{1}{2}}-q^{\frac{1}{2}}\right)^{2 g-1} \lambda^{Q} ， \\
& f_{\text {日 }}(q, \lambda)=\sum_{Q} \sum_{g \geq 0}\left(q \widehat{N}_{\square, g, Q}-\widehat{N}_{\boxminus, g, Q}+q^{-1} \widehat{N}_{\text {日, }, Q, Q}\right)\left(q^{-\frac{1}{2}}-q^{\frac{1}{2}}\right)^{2 g-1} \lambda^{Q} .
\end{aligned}
$$

One important consequence of (3.19) is that it encodes the structure of the large $N$ expansion (2.18). To show this, it is very convenient to transform the $f_{R}$ functions to the $\vec{k}$-basis. We then define:

$$
f_{\vec{k}}(q, \lambda)=\sum_{R} \chi_{R}(C(\vec{k})) f_{R}(q, \lambda)
$$

Notice that, in terms of these functions, the equation (2.15) reads

$$
G_{\vec{k}}^{(c)}(q, \lambda)=\sum_{n \mid \vec{k}} n^{|\vec{k}|-1} f_{\vec{k}_{1 / n}}\left(q^{n}, \lambda^{n}\right) .
$$

This implies that the functions $f_{\vec{k}}(q, \lambda)$ are given by the connected Green functions, corrected by some lower order terms that involve $f_{\vec{k}^{\prime}}$ with $\ell^{\prime} \leq \ell$. Since

$$
\frac{|C(\vec{k})|}{\ell !} G_{\vec{k}}^{(c)}(U)=\sum_{g=0}^{\infty} F_{g, \vec{k}}(\lambda) g_{s}^{2 g-2+|\vec{k}|}
$$

to prove that (3.19) explains the relations between the $N_{R, Q, s}$ observed in [3], one has to show that the right hand side of (3.28) has an expansion in $g_{s}$ of the form (3.29). 
The proof goes as follows. Define the following integers:

$$
n_{\vec{k}, g, Q}=\sum_{R} \chi_{R}(C(\vec{k})) \widehat{N}_{R, g, Q}
$$

Using again the expression (3.23), and (3.13)(3.17), it is easy to prove that the $f_{\vec{k}}(q, \lambda)$ have the following structure:

$$
f_{\vec{k}}(q, \lambda)=\left(\frac{\prod_{j}\left(q^{-\frac{j}{2}}-q^{\frac{j}{2}}\right)^{k_{j}}}{\left(q^{-\frac{1}{2}}-q^{\frac{1}{2}}\right)^{2}}\right) \sum_{Q} \sum_{g \geq 0} n_{\vec{k}, g, Q}\left(q^{-\frac{1}{2}}-q^{\frac{1}{2}}\right)^{2 g} \lambda^{Q} .
$$

We can now show that this target space prediction gives the $1 / N$ expansion $(2.18)$ for the connected vevs. Notice first that

$$
f_{\vec{k}}\left(q^{-1}, \lambda\right)=(-1)^{|k|} f_{\vec{k}}(q, \lambda)
$$

If we expand in $g_{s}$, the leading power comes from the overall fraction in (3.31):

$$
\frac{\prod_{j}\left(q^{\frac{j}{2}}-q^{-\frac{j}{2}}\right)^{k_{j}}}{\left(q^{\frac{1}{2}}-q^{-\frac{1}{2}}\right)^{2}}=g_{s}^{|\vec{k}|-2}+\cdots
$$

Since $f_{\vec{k}}(q, \lambda)$ has parity $(-1)^{|\vec{k}|}$ under $g_{s} \leftrightarrow-g_{s}$, the powers of $g_{s}$ in the expansion are $2 n+|\vec{k}|-2$, with $n \geq 0$. This is precisely the structure of (2.18). Using the definition of $\vec{k}_{1 / n}$, it is easy to see that the terms $f_{\vec{k}_{1 / n}}\left(q^{n}, \lambda^{n}\right)$ in $(3.28)$, for $n>1$, have the same perturbative expansion in $g_{s}$, and this finally shows that the structure of the expansion (2.18) is a consequence of (3.31). We conclude that the structure theorem (3.19) encodes all the relations between the integers $N_{R, Q, s}$ found in [3].

As a final remark, it is interesting to give the geometric interpretation of the integers defined in (3.30). If the symmetric group $S_{\ell}$ is acting on a manifold $M$, the fixed points of the action will be labeled by the conjugacy classes $C(\vec{k})$. We will denote them by $M^{\vec{k}}$. One can then consider the invariant part of the cohomology of $M$ under the Young symmetrizer $c_{R}$. The fixed point theorem then tells us that

$$
\chi\left(\mathbf{S}_{R}\left(H^{*}(M)\right)\right)=\sum_{\vec{k}} \frac{|C(\vec{k})|}{\ell !} \chi_{R}(C(\vec{k})) \chi\left(M^{\vec{k}}\right) .
$$

This is just the expression of the fact that if the group $G$ acts on $M$, the number of cohomology elements of $M$ in the representation $R$ of $G$ are given by the sum of cohomologies 
of subspaces of $M$ fixed by each element of $h \in G$, weighted by the character of $h$ in representation $R, \chi_{R}(h) /|G|$. In our context, $M=\mathcal{M}_{g, \ell}$, and the symmetry group acts by permutation of the boundaries. The integer $n_{\vec{k}, g, Q}$ is then interpreted as the Euler characteristic of the fixed point subset of $\mathcal{M}_{g, \ell}$ labeled by $\vec{k}=\left(k_{1}, k_{2}, \cdots\right)$. In this subset we have $k_{1}$ simple boundaries, $k_{2}$ double boundaries, and in general $k_{j}$ boundaries made up of $j$ boundaries that have collided. Therefore, this geometric configuration has effectively $h=\sum_{j} k_{j}$ boundaries, as in the $1 / N$ expansion (2.18).

\section{Links and topological strings}

\subsection{Generalization to links}

In this section, we will generalize the construction of [2] [3] and of the above sections to links. Let's consider a link $\mathcal{L}$ with $L$ components $\gamma_{\alpha}, \alpha=1, \cdots, L$. As explained in section 2 , the BPS states will be classified now by the quantum numbers $Q$, the spin $s$ and the representations $R_{1}, \cdots, R_{L}$ associated to the D2 branes ending on the Lagrangian submanifolds $D_{\alpha}$. The integers associated to these BPS states will be denoted by $N_{\left(R_{1}, \cdots, R_{L}\right) ; Q, s}$. The free energy is then given by

$$
F\left(V_{\alpha}\right)=\sum_{n=1}^{\infty} \sum_{R_{1}, \cdots, R_{L}} \sum_{Q, s} \frac{N_{\left(R_{1}, \cdots, R_{L}\right) ; Q, s}}{q^{\frac{n}{2}}-q^{-\frac{n}{2}}} \frac{q^{n s} \lambda^{n Q}}{n} \prod_{\alpha=1}^{L} \operatorname{Tr}_{R_{\alpha}} V_{\alpha}^{n}
$$

The main conjecture is then that

$$
\left\langle Z\left(U_{1}, \cdots, U_{L} ; V_{1}, \cdots, V_{L}\right)\right\rangle=\left\langle\exp \left[\sum_{\alpha=1}^{L} \sum_{n=1}^{\infty} \frac{1}{n} \operatorname{Tr} U_{\alpha}^{n} \operatorname{Tr} V_{\alpha}^{n}\right]\right\rangle=\exp F\left(V_{\alpha}\right)
$$

The generalization of (2.15) is straightforward. The basis of operators is labeled now by vectors $\vec{k}^{(\alpha)}, \alpha=1, \cdots, L$, and we define the vev $G_{\vec{k}^{(1)}, \cdots, \vec{k}^{(L)}}\left(U_{1}, \cdots, U_{L}\right)$ as follows:

$$
G_{\vec{k}^{(1)}, \cdots, \vec{k}^{(L)}}\left(U_{1}, \cdots, U_{L}\right)=\left\langle\prod_{\alpha=1}^{L} \Upsilon_{\vec{k}^{(\alpha)}}\left(U_{\alpha}\right)\right\rangle
$$

where $U_{\alpha}$ is the holonomy around $\mathcal{K}_{\alpha}$. Using Frobenius formula, we can obtain the representation basis for the Wilson loop operators:

$$
\operatorname{Tr}_{R_{1}}\left(U_{1}\right) \cdots \operatorname{Tr}_{R_{L}}\left(U_{L}\right)=\prod_{\alpha=1}^{L} \chi_{R_{\alpha}}\left(C\left(\vec{k}^{(\alpha)}\right)\right) \Upsilon_{\vec{k}^{(\alpha)}}\left(U_{\alpha}\right)
$$


The connected vevs are defined, as usual, by taking the logarithm of the generating function. More precisely, associate to any $\vec{k}$ the polynomial $p_{\vec{k}}(x)=\prod_{j} x_{j}^{k_{j}}$ in the variables $x_{1}, x_{2}, \ldots$ We then define the "connected" coefficients $a_{\vec{k}^{(1)}, \ldots, \vec{k}^{(L)}}$ as follows:

$$
\begin{gathered}
\log \left(1+\sum_{L} \sum_{\vec{k}^{(\alpha)}} a_{\vec{k}^{(1)}, \ldots, \vec{k}^{(L)}} \prod_{\alpha=1}^{L} \frac{\left|C\left(\vec{k}^{(\alpha)}\right)\right|}{\ell_{\alpha} !} p_{\vec{k}^{(\alpha)}}\left(x^{(\alpha)}\right)\right)= \\
\sum_{L} \sum_{\vec{k}^{(\alpha)}} a_{\vec{k}^{(1)}, \ldots, \vec{k}^{(L)}}^{(c)} \prod_{\alpha=1}^{L} \frac{\left|C\left(\vec{k}^{(\alpha)}\right)\right|}{\ell !} p_{\vec{k}^{(\alpha)}}\left(x^{(\alpha)}\right) .
\end{gathered}
$$

It is again convenient to group the numbers $N_{\left(R_{1}, \cdots, R_{L}\right) ; Q, s}$ into the polynomials

$$
f_{\left(R_{1}, R_{2}, \cdots, R_{L}\right)}(q, \lambda)=\sum_{Q, s} \frac{N_{\left(R_{1}, \cdots, R_{L}\right) ; Q, s}}{q^{\frac{1}{2}}-q^{-\frac{1}{2}}} q^{s} \lambda^{Q}
$$

A simple generalization of the arguments of [3] gives the following relation for links:

$$
G_{\vec{k}^{(1)}, \cdots, \vec{k}^{(L)}}^{(c)}\left(U_{1}, \cdots, U_{L}\right)=\sum_{n \mid \vec{k}^{(\alpha)}} n^{\sum_{\alpha}\left|\vec{k}^{(\alpha)}\right|-1} \sum_{R_{1}, \cdots, R_{L}} \prod_{\alpha=1}^{L} \chi_{R_{\alpha}}\left(C\left(\vec{k}_{1 / n}^{(\alpha)}\right)\right) f_{\left(R_{1}, \cdots, R_{L}\right)}(q, \lambda) .
$$

In the first sum, the vector $\vec{k}_{1 / n}$ is defined as in (2.15). In (4.7), the $n$ have to divide all the vectors $\vec{k}^{(\alpha)}, \alpha=1, \cdots, L$. As in [3], it is easy to see that, given the connected vevs, the equation (4.7) determines the generating functions $f_{\left(R_{1}, \cdots, R_{L}\right)}(q, \lambda)$ in a unique way by a recursive procedure. For every vector $\left(\ell_{1}, \cdots, \ell_{L}\right)$ (which specifies the "order") we can solve for the $f$ 's in terms of the connected vevs and the $f$ 's of lower order (i.e. with $\left.\ell_{\alpha}^{\prime} \leq \ell_{\alpha}\right)$.

Let us now give some examples of this procedure. The simplest case of (4.7) is for $R_{1}=\cdots=R_{L}=\square$. In this case, one also has $\vec{k}^{(1)}=\cdots \vec{k}^{(L)}=(1,0, \cdots)$, and

$$
f_{(\square, \cdots, \square)}=G_{(1,0, \cdots), \cdots,(1,0, \cdots)}^{(c)}\left(U_{1}, \cdots, U_{L}\right) .
$$

Notice that the connected vacuum expectation value contains information also about the vevs for the components of the link, and for all the possible sublinks that can be formed with these components. For example, for links with two components, one has

$$
f_{(\square, \square)}=\left\langle\operatorname{Tr} U_{1} \operatorname{Tr} U_{2}\right\rangle-\left\langle\operatorname{Tr} U_{1}\right\rangle\left\langle\operatorname{Tr} U_{2}\right\rangle
$$

where the second piece is the right hand side is the product of the vevs for the two knots, $\mathcal{K}_{1}$ and $\mathcal{K}_{2}$, that form the link. 
Let us now focus on links with two components. The next order is specified by $\left(\ell_{1}, \ell_{2}\right)=(2,1)$ and $\left(\ell_{1}, \ell_{2}\right)=(1,2)$. We have, for example,

$$
\begin{aligned}
f_{(\square, \square)} & =\frac{1}{2}\left(G_{(2,0, \cdots),(1,0, \cdots)}^{(c)}+G_{(0,1,0, \cdots),(1,0, \cdots)}^{(c)}\right), \\
f_{(日, \square)} & =\frac{1}{2}\left(G_{(2,0, \cdots),(1,0, \cdots)}^{(c)}-G_{(0,1,0, \cdots),(1,0, \cdots)}^{(c)}\right) .
\end{aligned}
$$

Of course, for $\left(\ell_{1}, \ell_{2}\right)=(1,2)$ we have the same equations but with the labels exchanged.

The next level is labeled by $(1,3),(3,1)$ and $(2,2)$. For $(3,1)$, (4.7) gives:

$$
\begin{aligned}
& f_{(\text {س, 口) }}=\frac{1}{6} G_{(3,0, \cdots),(1,0, \cdots)}^{(c)}+\frac{1}{2} G_{(1,1,0, \cdots),(1,0, \cdots)}^{(c)}+\frac{1}{3} G_{(0,0,1, \cdots),(1,0, \cdots)}^{(c)}, \\
& f_{(\boxplus, \square)}=\frac{1}{3}\left(G_{(3,0, \cdots),(1,0, \cdots)}^{(c)}-G_{(0,0,1, \cdots),(1,0, \cdots)}^{(c)}\right), \\
& f_{\text {(日, 口) }}=\frac{1}{6} G_{(3,0, \cdots),(1,0, \cdots)}^{(c)}-\frac{1}{2} G_{(1,1,0, \cdots),(1,0, \cdots)}^{(c)}+\frac{1}{3} G_{(0,0,1, \cdots),(1,0, \cdots)}^{(c)} .
\end{aligned}
$$

The equations for the order $(1,2)$ are again obtained by exchanging the labels. Finally, for $(2,2)$ we obtain

$$
\begin{aligned}
& f_{(\varpi, \varpi)}(q, \lambda)=\frac{1}{4}\left(G_{(2,0, \cdots),(2,0, \cdots)}^{(c)}+G_{(0,1,0, \cdots),(2,0, \cdots)}^{(c)}+G_{(2,0, \cdots),(0,1, \cdots)}^{(c)}+G_{(0,1, \cdots),(0,1, \cdots)}^{(c)}\right) \\
& -\frac{1}{2} f_{(\square, \square)}\left(q^{2}, \lambda^{2}\right) \\
& f_{(\text {口, 日) }}(q, \lambda)=\frac{1}{4}\left(G_{(2,0, \cdots),(2,0, \cdots)}^{(c)}+G_{(0,1,0, \cdots),(2,0, \cdots)}^{(c)}-G_{(2,0, \cdots),(0,1, \cdots)}^{(c)}-G_{(0,1, \cdots),(0,1, \cdots)}^{(c)}\right) \\
& +\frac{1}{2} f_{(\square, \square)}\left(q^{2}, \lambda^{2}\right) \\
& f_{\text {(日,田) }}(q, \lambda)=\frac{1}{4}\left(G_{(2,0, \cdots),(2,0, \cdots)}^{(c)}-G_{(0,1,0, \cdots),(2,0, \cdots)}^{(c)}+G_{(2,0, \cdots),(0,1, \cdots)}^{(c)}-G_{(0,1, \cdots),(0,1, \cdots)}^{(c)}\right) \\
& +\frac{1}{2} f_{(\square, \square)}\left(t^{2}, \lambda^{2}\right) \\
& f_{\text {(日, 日 })}(q, \lambda)=\frac{1}{4}\left(G_{(2,0, \cdots),(2,0, \cdots)}^{(c)}-G_{(0,1,0, \cdots),(2,0, \cdots)}^{(c)}-G_{(2,0, \cdots),(0,1, \cdots)}^{(c)}+G_{(0,1, \cdots),(0,1, \cdots)}^{(c)}\right) \\
& -\frac{1}{2} f_{(\square, \square)}\left(q^{2}, \lambda^{2}\right) \text {. }
\end{aligned}
$$

\subsection{D-branes and links}

A straightforward generalization of the arguments in section 2 goes as follows. If we have a link of $L$ components, the corresponding Lagrangian submanifold $D=\cup D_{\alpha}$ after the transition will have $b_{1}=L$. We will denote by $Y_{\alpha}$ the nontrivial one-cycles of $D$, with 
$\alpha=1, \cdots, L$. After performing the analytic continuation, the $1 / N$ expansion (2.7) can be written as

$$
F\left(V_{\alpha}\right)=\sum_{\vec{k}^{(1)}, \ldots, \vec{k}^{(L)}} \sum_{g=0}^{\infty} g_{s}^{2 g-2+\sum_{\alpha=1}^{L}\left|\vec{k}^{(\alpha)}\right|} F_{g ; \vec{k}^{(1)}, \ldots, \vec{k}^{(L)}}(\lambda) \Upsilon_{\vec{k}^{(\alpha)}}\left(V_{\alpha}\right)
$$

where $\vec{k}^{\alpha}$ denotes the number of holes, together with their wrappings which end on $D_{\alpha}$.

The D-brane derivation of this expansion proceeds as in section 3. We have to consider Riemann surfaces with $\ell=\sum_{\alpha=1}^{L} \ell_{\alpha}$ boundaries in such a way that $\ell_{\alpha}$ boundaries end on $Y_{\alpha}$. The relevant symmetry group is now $S_{\ell_{1}} \times \cdots \times S_{\ell_{L}}$, and the starting point is again (3.1). Projecting onto symmetric configurations under the action of $\prod_{\alpha=1}^{L} S_{\ell_{\alpha}}$, one finds:

$$
\sum_{R_{1}, \cdots, R_{L}}\left(\mathbf{S}_{R_{1}}\left(F^{\ell_{1}}\right) \otimes \cdots \otimes \mathbf{S}_{R_{L}}\left(F^{\ell_{L}}\right)\right) \otimes \mathbf{S}_{R_{1}, \cdots, R_{L}}\left(H^{*}\left(\left(\mathbf{S}^{1}\right)^{\ell-1}\right) \otimes H^{*}\left(\mathcal{M}_{g, \ell}\right)\right) .
$$

The first factor is simply the tensor product of the irreducible representations $R_{1}, \cdots, R_{L}$ of $S U(N)$, while the second factor can be further decomposed as

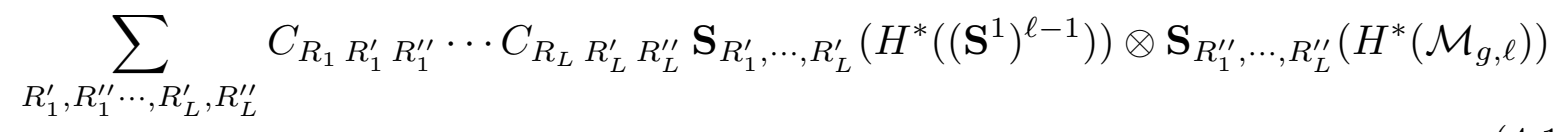

The generating functions associated to $\mathbf{S}_{R_{1}^{\prime}, \cdots, R_{L}^{\prime}}\left(H^{*}\left(\left(\mathbf{S}^{1}\right)^{\ell-1}\right)\right)$ are given by a straightforward generalization of the arguments for the $L=1$ case. The result is:

$$
\operatorname{Tr}_{\mathbf{S}_{R_{1}, \cdots, R_{L}}\left(H^{*}\left(\left(\mathbf{S}^{1}\right)^{\ell-1}\right)\right)}(-1)^{F} q^{s}=\left(q^{-\frac{1}{2}}-q^{\frac{1}{2}}\right)^{L-1} \prod_{\alpha=1}^{L} S_{R_{\alpha}}(q)
$$

The new integer invariants will then be, in the case of links,

$$
\widehat{N}_{\left(R_{1}, \cdots, R_{L}\right), g, Q}=\chi\left(\mathbf{S}_{R_{1}, \cdots, R_{L}}\left(H^{*}\left(\mathcal{M}_{g, \ell}\right)\right)\right),
$$

and the generating functions (4.6) have the structure:

$$
\begin{aligned}
& f_{\left(R_{1}, \cdots, R_{L}\right)}(q, \lambda)= \\
& \left(q^{-\frac{1}{2}}-q^{\frac{1}{2}}\right)^{L-2} \sum_{g \geq 0} \sum_{Q} \sum_{R_{1}^{\prime}, R_{1}^{\prime \prime} \cdots, R_{L}^{\prime}, R_{L}^{\prime \prime}} \prod_{\alpha=1}^{L} C_{R_{\alpha} R_{\alpha}^{\prime} R_{\alpha}^{\prime \prime}} S_{R_{\alpha}^{\prime}}(q) \widehat{N}_{\left(R_{1}^{\prime \prime}, \cdots, R_{L}^{\prime \prime}\right), g, Q}\left(q^{-\frac{1}{2}}-q^{\frac{1}{2}}\right)^{2 g} \lambda^{Q} .
\end{aligned}
$$


We can also define

$$
\widehat{f}_{\left(R_{1}, \cdots, R_{L}\right)}(q, \lambda)=\left(q^{-\frac{1}{2}}-q^{\frac{1}{2}}\right)^{L-2} \sum_{g \geq 0} \sum_{Q} \widehat{N}_{\left(R_{1}, \cdots, R_{L}\right), g, Q}\left(q^{-\frac{1}{2}}-q^{\frac{1}{2}}\right)^{2 g} \lambda^{Q}
$$

We then have the relation:

$$
f_{\left(R_{1}, \cdots, R_{L}\right)}(q, \lambda)=\sum_{R_{1}^{\prime}, \cdots, R_{L}^{\prime}} M_{R_{1}, \cdots, R_{L} ; R_{1}^{\prime}, \cdots, R_{L}^{\prime}}(q) \widehat{f}_{\left(R_{1}, \cdots, R_{L}\right)}(q, \lambda)
$$

where the matrix $M_{R_{1}, \cdots, R_{L} ; R_{1}^{\prime}, \cdots, R_{L}^{\prime}}(q)$ is given by

$$
\begin{aligned}
M_{R_{1}, \cdots, R_{L} ; R_{1}^{\prime}, \cdots, R_{L}^{\prime}}(q) & =\sum_{R_{1}^{\prime \prime}, \cdots, R_{L}^{\prime \prime}} \prod_{\alpha=1}^{L} C_{R_{\alpha} R_{\alpha}^{\prime} R_{\alpha}^{\prime \prime}} S_{R_{\alpha}^{\prime \prime}}(q) \\
& =\sum_{\vec{k}^{(1)}, \cdots, \vec{k}^{(L)}} \prod_{\alpha=1}^{L} \frac{\left|C\left(\vec{k}^{(\alpha)}\right)\right|}{\ell_{\alpha} !} \chi_{R_{\alpha}}\left(C\left(\vec{k}^{(\alpha)}\right)\right) \chi_{R_{\alpha}^{\prime}}\left(C\left(\vec{k}^{(\alpha)}\right)\right) P_{\vec{k}^{(\alpha)}}(q) .
\end{aligned}
$$

The generalization of (2.17) to the case of links is

$$
N_{\left(R_{1}, \cdots, R_{L}\right) ; Q, s}=(-1)^{\sum_{\alpha=1}^{L} \ell_{\alpha}-1} N_{\left(R_{1}^{t}, \cdots, R_{L}^{t}\right) ; Q,-s},
$$

or, equivalently,

$$
f_{\left(R_{1}, \cdots, R_{L}\right)}\left(t^{-1}, \lambda\right)=(-1)^{\sum_{\alpha=1}^{L} \ell_{\alpha}} f_{\left(R_{1}^{t}, \cdots, R_{L}^{t}\right)}(t, \lambda)
$$

which are a consequence of (3.12) and (4.18). Again, we can obtain the structure of the generating functions in the $\vec{k}$ basis, by defining

$$
f_{\vec{k}^{(1)}, \cdots, \vec{k}^{(L)}}(q, \lambda)=\sum_{R_{1}, \cdots, R_{L}} \prod_{\alpha=1}^{L} \chi_{R_{\alpha}}\left(C\left(\vec{k}^{(\alpha)}\right)\right) f_{\left(R_{1}, \cdots, R_{L}\right)}(q, \lambda) .
$$

and

$$
n_{\left(\vec{k}^{(1)}, \cdots, \vec{k}^{(L)}\right), g, Q}=\sum_{R_{1}, \cdots, R_{L}} \prod_{\alpha=1}^{L} \chi_{R_{\alpha}}\left(C\left(\vec{k}^{(\alpha)}\right)\right) \widehat{N}_{\left(R_{1}, \cdots, R_{L}\right), g, Q} .
$$

A straightforward generalization of the argument in the case of knots shows that

$$
f_{\vec{k}^{(1)}, \cdots, \vec{k}^{(L)}}(q, \lambda)=\left(\frac{\prod_{j}\left(q^{-\frac{j}{2}}-q^{\frac{j}{2}}\right)^{\sum_{\alpha=1}^{L} k_{j}^{(\alpha)}}}{\left(q^{-\frac{1}{2}}-q^{\frac{1}{2}}\right)^{2}}\right) \sum_{Q} \sum_{g \geq 0} n_{\left(\vec{k}^{(1)}, \cdots, \vec{k}^{(L)}\right), g, Q}\left(q^{-\frac{1}{2}}-q^{\frac{1}{2}}\right)^{2 g} \lambda^{Q} .
$$

and this leads to the $1 / N$ expansion (4.13). 


\subsection{Links in Chern-Simons theory}

In the previous section we have generalized the results of [2] [3] and section 3 to the case of links in a rather straightforward way. The subtlety with links has to do rather with the computation of the vevs in Chern-Simons theory. It turns out that there are some ambiguities involved in this computation, and as we will see the appropriate definition of the vev is different from the standard one in knot theory.

A good starting point to address this issue is the perturbative analysis of vevs in ChernSimons theory [16] 17]. Let us consider a link $\mathcal{L}$ with $L$ components $\mathcal{K}_{\alpha}, \alpha=1, \cdots, L$, and let's assume for simplicity that all the components carry the fundamental representation. In Chern-Simons theory the components of a link have to be framed and the resulting invariants are invariants of framed links. We will assume that the components are in the vertical framing, which is very natural when we define the invariant in terms of a plane projection of the link [18]. Vevs computed in the vertical framing are not ambient isotopy invariants, but rather regular isotopy invariant (this means that they are not invariant under Reidemeister type I moves). However, a perturbative analysis of the vev [16] [17] shows that the corrected quantity

$$
\exp \left[-2 \pi i w(\mathcal{L}) h_{\square}\right]\langle W(\mathcal{L})\rangle^{\mathrm{vf}},
$$

where $w(\mathcal{L})$ is the writhe of the planar projection of the link, is an ambient isotopy invariant and in particular gives the unnormalized HOMFLY polynomial of the link. In this equation, $h_{R}$ is the conformal weight of the WZW model for the representation $R$. For the fundamental representation it is given by $h_{\square}=(N-1 / N) /(2(k+N))$. The key thing here (which is familiar from the construction of the Kauffman bracket) is that the variation of $\langle W(\mathcal{L})\rangle^{\mathrm{vf}}$ under type I moves is compensated by the variation of the writhe, in such a way that the product is a topological invariant. Notice that the writhe of the link is given by:

$$
w(\mathcal{L})=\sum_{\alpha=1}^{L} w\left(\mathcal{K}_{\alpha}\right)+2 \operatorname{lk}(\mathcal{L}),
$$

where $\operatorname{lk}(\mathcal{L})$, the total linking number of $\mathcal{L}$, is defined as:

$$
\operatorname{lk}(\mathcal{L})=\sum_{\alpha<\beta} \operatorname{lk}\left(\mathcal{K}_{\alpha}, \mathcal{K}_{\beta}\right),
$$


and $\operatorname{lk}\left(\mathcal{K}_{\alpha}, \mathcal{K}_{\beta}\right)$ is the linking number of the two components $\alpha, \beta$. These linking numbers are ambient isotopy invariants of the link. Therefore, in order to obtain a welldefined invariant of links, it would have been enough to introduce the correction factor $\exp \left[-2 \pi i \sum_{\alpha} w\left(\mathcal{K}_{\alpha}\right) h_{\square}\right]$. From a topological point of view, there is an important advantage in defining the invariant as in (4.27): with that definition, all the crossings in the link are treated in the same way, without taking into account if they belong to the same component or to different components of the link (for a related discussion, see [19]). This is why the usual HOMFLY polynomial satisfies a universal skein relation.

The main conclusion of this analysis is that, in order to obtain the usual knot invariants, one has to correct the field theory vev in two ways. First, as for knots, we have to include a non-topological factor to take into account the framing of the components. The resulting vev is in the standard framing and will be denoted by $\langle W(\mathcal{L})\rangle_{\mathrm{sf}}$ :

$$
\langle W(\mathcal{L})\rangle^{\mathrm{sf}}=\exp \left[-2 \pi i \sum_{\alpha} w\left(\mathcal{K}_{\alpha}\right) h_{\square}\right]\langle W(\mathcal{L})\rangle^{\mathrm{vf}}
$$

The second factor is topological and depends on the linking numbers of the components:

$$
\exp \left[-4 \pi i h_{\square} \sum_{\alpha<\beta} \operatorname{lk}\left(\mathcal{K}_{\alpha}, \mathcal{K}_{\beta}\right)\right]
$$

Notice that (4.31) can be written as:

$$
\exp \left[\frac{2 \pi i}{N(k+N)} \sum_{\alpha<\beta} \operatorname{lk}\left(\mathcal{K}_{\alpha}, \mathcal{K}_{\beta}\right)\right] \lambda^{-\sum_{\alpha<\beta} \operatorname{lk}\left(\mathcal{K}_{\alpha}, \mathcal{K}_{\beta}\right)}
$$

and the first factor guarantees that the corrected vev is a polynomial in $t$ and $\lambda$. However, this correction has some unpleasant features. It can be easily seen that the second factor spoils the structure of the $1 / N$ expansion (2.18) of the field-theory vev. This seems to be a problem in order to find a string interpretation of the Chern-Simons vevs. On the other hand, the string interpretation makes clear that the appropriate vevs should be functions of $t$ and $\lambda$, so the vev still has to be corrected with the first factor of (4.32) in order to compare it to the string predictions. Therefore, we propose that the relevant vev that is needed for comparison with the string predictions is

$$
\exp \left[\frac{2 \pi i}{N(k+N)} \sum_{\alpha<\beta} \operatorname{lk}\left(\mathcal{K}_{\alpha}, \mathcal{K}_{\beta}\right)\right]\langle W(\mathcal{L})\rangle^{\mathrm{sf}}
$$


This means, in particular, that the relevant link polynomial is not the HOMFLY polynomial $P_{\mathcal{L}}(t, \lambda)$, but rather

$$
\lambda^{\operatorname{lk}(\mathcal{L})} P_{\mathcal{L}}(t, \lambda)
$$

As we will see, this is crucial in order to find agreement with the string predictions.

In the discussion above, we have assumed that all the components of the links were in the fundamental representation. We will now consider the general case in which the components are in arbitrary irreducible representations of $S U(N)$. To do that, we will introduce some notation. Let $R$ be an irreducible representation, and let $\Lambda$ be the highest weight. If we denote by $\lambda_{i}, i=1, \cdots, N-1$ the fundamental weights of $S U(N)$, we can always write

$$
\Lambda=\sum_{i=1}^{N-1} a_{i} \lambda_{i}
$$

where the $a_{i}$ are nonnegative integers. We can associate to $R$ a Young diagram in the usual way, with $\ell=\sum_{i} i a_{i}$ boxes. Consider the following integer associated to $\Lambda$, which will be useful later on:

$$
\kappa_{\Lambda}=\sum_{i}\left(i a_{i}^{2}-i^{2} a_{i}\right)+2 \sum_{i<j} i a_{i} a_{j} .
$$

The conformal weight $h_{R}$ is then given by:

$$
h_{R}=\frac{\Lambda \cdot(\Lambda+2 \rho)}{2(k+N)},
$$

where $\rho$ is the Weyl vector (i.e., the sum of the fundamental weights). Notice that

$$
\Lambda \cdot(\Lambda+2 \rho)=N \ell-\frac{\ell^{2}}{N}+\kappa_{\Lambda}
$$

Let's then consider a "multicoloured" link with $L$ components, with $\mathcal{K}_{\alpha}$ in the representation $R_{\alpha}$, for $\alpha=1, \cdots, L$. The number of boxes in the Young tableau associated to $R_{\alpha}$ is denoted by $\ell_{\alpha}$. The generalization of (4.30) to this situation is simply

$$
\langle W(\mathcal{L})\rangle^{\mathrm{sf}}=\exp \left[-2 \pi i \sum_{\alpha} w\left(\mathcal{K}_{\alpha}\right) h_{R_{\alpha}}\right]\langle W(\mathcal{L})\rangle^{\mathrm{vf}}
$$

We also propose that the corrected vev which extends (4.33) to the general case is

$$
\exp \left[\frac{2 \pi i}{N(k+N)} \sum_{\alpha<\beta} \operatorname{lk}\left(\mathcal{K}_{\alpha}, \mathcal{K}_{\beta}\right) \ell_{\alpha} \ell_{\beta}\right]\langle W(\mathcal{L})\rangle^{s \mathrm{f}} .
$$

This choice is motivated by the structure of the knot operators for torus knots and links, that will be reviewed later. (4.40) is the minimal correction that makes the resulting object a function of $t^{ \pm 1 / 2}$ and $\lambda^{ \pm 1 / 2}$, and it can be easily checked that it preserves the structure of the $1 / N$ expansion. 


\subsection{Rank-level duality for links}

Rank-level duality of WZW models [20] implies a certain number of identities for Chern-Simons vevs [21], which essentially relate the vev of a Wilson loop in the representation $R$ with the vev in the transposed representation. In this section we will see that these identities are in fact closely related to the relation (4.23). The comparison to the results of [21] involves the extra factor (4.40) in an interesting way, and provides a further consistency check of our procedure.

Using (4.23) and (4.7) one can obtain the following relation for the vevs of a product of Wilson loops in the representation basis:

$$
\left\langle\operatorname{Tr}_{R_{1}}\left(U_{1}\right) \cdots \operatorname{Tr}_{R_{L}}\left(U_{L}\right)\right\rangle\left(q^{-1}, \lambda\right)=(-1)^{\ell}\left\langle\operatorname{Tr}_{R_{1}^{t}}\left(U_{1}\right) \cdots \operatorname{Tr}_{R_{L}^{t}}\left(U_{L}\right)\right\rangle(q, \lambda),
$$

where $\ell=\sum_{\alpha} \ell_{\alpha}$. This relation is in fact a consequence of the usual $1 / N$ expansion of the Wilson loops in Chern-Simons theory, and of the fact that the vevs can be written in terms of the variables $q, \lambda$. Therefore, for this relation to be true it is crucial to introduce the correction factors (4.40). To make contact with the rank-level duality relations obtained in [21], we have to go to the vertical framing. The vevs in the vertical framing are related to our corrected vevs through (4.30)(4.40):

$$
\begin{aligned}
& \left\langle\operatorname{Tr}_{R_{1}}\left(U_{1}\right) \cdots \operatorname{Tr}_{R_{L}}\left(U_{L}\right)\right\rangle^{\mathrm{vf}}= \\
& \exp \left[-2 \pi i \sum_{\alpha} w\left(\mathcal{K}_{\alpha}\right) h_{R_{\alpha}}-\frac{2 \pi i}{N(k+N)} \sum_{\alpha<\beta} \operatorname{lk}\left(\mathcal{K}_{\alpha}, \mathcal{K}_{\beta}\right) \ell_{\alpha} \ell_{\beta}\right]\left\langle\operatorname{Tr}_{R_{1}}\left(U_{1}\right) \cdots \operatorname{Tr}_{R_{L}}\left(U_{L}\right)\right\rangle .
\end{aligned}
$$

Now we use the fact that taking the mirror image $\widetilde{U}$ of a Wilson line $U$ is equivalent to complex conjugation and sends $q, \lambda \rightarrow q^{-1}, \lambda^{-1}$. Finally, using that [21]

$$
\left.h_{R_{\alpha}}\right|_{S U(N)_{k}}+\left.h_{R_{\alpha}^{t}}\right|_{S U(k)_{N}}=\frac{\ell_{\alpha}}{2}-\frac{\ell_{\alpha}^{2}}{2 N k},
$$

we obtain

$$
\begin{aligned}
& \left\langle\operatorname{Tr}_{R_{1}}\left(U_{1}\right) \cdots \operatorname{Tr}_{R_{L}}\left(U_{L}\right)\right\rangle_{S U(N)_{k}}^{\mathrm{vf}}= \\
& \exp \left[\pi i \sum_{\alpha} w\left(\mathcal{K}_{\alpha}\right) \ell_{\alpha}-\frac{2 \pi i}{N(k+N)} \sum_{\alpha, \beta} \operatorname{lk}\left(\mathcal{K}_{\alpha}, \mathcal{K}_{\beta}\right) \ell_{\alpha} \ell_{\beta}\right]\left\langle\operatorname{Tr}_{R_{1}^{t}}\left(\widetilde{U}_{1}\right) \cdots \operatorname{Tr}_{R_{L}^{t}}\left(\widetilde{U}_{L}\right)\right\rangle_{S U(k)_{N}}^{\mathrm{vf}},
\end{aligned}
$$

where the self-linking number is $\operatorname{lk}\left(\mathcal{K}_{\alpha}, \mathcal{K}_{\alpha}\right)=w\left(\mathcal{K}_{\alpha}\right)$. To write the final result, we have also taken into account that the vev of $\operatorname{Tr}_{R_{1}}\left(U_{1}\right) \cdots \operatorname{Tr}_{R_{L}}\left(U_{L}\right)$ has the monodromy $(-1)^{\ell}$ under $\lambda \rightarrow \mathrm{e}^{2 \pi i} \lambda$. We then see that the rank-level duality relations of [21] are a consequence of both the structure of the $1 / N$ expansion (or its D-brane version (4.26)) and the monodromy of the vevs under $\lambda \rightarrow \mathrm{e}^{2 \pi i} \lambda$. 


\subsection{Polynomial invariants for torus links}

To obtain explicit results for the generating functions $f_{\left(R_{1}, \cdots, R_{L}\right)}$, we have to compute vevs of general products of Wilson loops. In general these computations are technically difficult, but if the components of the links are torus knots, one can use the formalism of knot operators developed in [22]. Torus knots are labeled by two coprime integers $(n, m)$, which correspond to winding numbers around the two non-contractible cycles of the torus. Wilson loops corresponding to a torus knot $(n, m)$, and in an irreducible representation of highest weight $\Lambda$, are represented by the operator $W_{\Lambda}^{(n, m)}$. This operator acts on the Hilbert space of Chern-Simons gauge theory on a torus. It has been shown in 223 that this space has an orthonormal basis $|p\rangle$ labeled by weights $p$ in the fundamental chamber of the weight lattice of $S U(N), \mathcal{F}_{l}$, where $l=k+N$. We take as representatives of $p$ the ones of the form $p=\sum_{i} p_{i} \lambda_{i}$, with $p_{i}>0$ and $\sum_{i} p_{i}<l$. The vacuum is the state $|\rho\rangle$, where $\rho$ is the Weyl vector. The action of the loop operator on a state $|p\rangle$ is given by [22]:

$$
W_{\Lambda}^{(n, m)}|p\rangle=\sum_{\mu \in M_{\Lambda}} \exp \left[-i \pi \mu^{2} \frac{n m}{k+N}-2 \pi i \frac{m}{k+N} p \cdot \mu\right]|p+n \mu\rangle .
$$

If we act on the vacuum with $L$ knot operators $\left(n_{1}, m_{1}\right), \cdots,\left(n_{L}, m_{L}\right)$, with representations labeled by $\Lambda_{1}, \cdots, \Lambda_{L}$, we will create a link whose $\alpha$-th component is a torus knot labeled by $\left(n_{\alpha}, m_{\alpha}\right)$ and representation $\Lambda_{\alpha}$ [19]. The resulting state can be computed from (4.45), and is given by

$$
\begin{aligned}
& \left(\prod_{\alpha=1}^{L} W_{\Lambda_{\alpha}}^{\left(n_{\alpha}, m_{\alpha}\right)}\right)|\rho\rangle=\sum_{\mu^{(\alpha)} \in M_{\Lambda_{\alpha}}} \exp \left[-\frac{2 \pi i}{k+N}\left(\sum_{\alpha<\beta} n_{\alpha} m_{\beta} \mu^{\alpha} \cdot \mu^{\beta}\right)\right] \\
& \times \exp \left[-\frac{i \pi}{k+N}\left(\sum_{\alpha} n_{\alpha} m_{\alpha}\left(\mu^{(\alpha)}\right)^{2}\right)-\frac{2 \pi i}{k+N} \rho \cdot\left(\sum_{\alpha} m_{\alpha} \mu^{(\alpha)}\right)\right]\left|\rho+\sum_{\alpha} n_{\alpha} \mu^{(\alpha)}\right\rangle .
\end{aligned}
$$

The link that has been constructed lives on the surface of a solid torus. To compute the vev of the product of Wilson loops when this link is in $\mathbf{S}^{3}$, we have to perform an $S$ transformation. The final expression for the vev, without including any correction factor, is [22] 19]

$$
\frac{\left\langle\rho\left|S \prod_{\alpha=1}^{L} W_{\Lambda_{\alpha}}^{\left(n_{\alpha}, m_{\alpha}\right)}\right| \rho\right\rangle}{\langle\rho|S| \rho\rangle} .
$$

As explained in [24], the inner products that appear in (4.47) can be computed as follows. The weight $\rho+\sum_{\alpha} n_{\alpha} \mu^{(\alpha)}$ is not necessarily in the fundamental chamber, but it will have 
a representative in it obtained by the action of an element of the Weyl group, say $w$. If this representative has a vanishing component in the Dynkin basis, then the corresponding state will be zero, due to the antisymmetry of the wave function under Weyl reflections. If this is not the case, we will be able to write the representative as $\rho+\mu_{\left(n_{1}, \cdots, n_{\alpha}\right)}$, where $\mu_{\left(n_{1}, \cdots, n_{\alpha}\right)}$ is a weight of nonnegative components. Using Weyl formula and the explicit expression for the $S$-operator, we can write

$$
\frac{\left\langle\rho|S| \rho+\sum_{\alpha} n_{\alpha} \mu^{(\alpha)}\right\rangle}{\langle\rho|S| \rho\rangle}=\epsilon(w) \operatorname{ch}_{\mu_{\left(n_{1}, \cdots, n_{\alpha}\right)}}\left[-\frac{2 \pi i}{k+N} \rho\right] .
$$

This solves in principle the problem of computing the vev (4.47).

We can now use these results to motivate (4.33). First notice that the exponential in (4.46) can not be written in terms of $t^{ \pm 1 / 2}, \lambda^{ \pm 1 / 2}$. This can be seen as follows. Let's denote by $\mu_{i}, i=1, \cdots, N$ the weights of the fundamental representation of $S U(N)$, and let $\Lambda$ be the highest weight of an irreducible representation of $S U(N)$ whose Young diagram contains $\ell$ boxes. It was shown in [3] that the weights in $M_{\Lambda}$ can be always written as

$$
k_{1} \mu_{i_{1}}+\cdots+k_{r} \mu_{i_{r}}, 1 \leq i_{1}<\ldots<i_{r} \leq N
$$

where $\left(k_{\lambda}\right)=\left(k_{1}, \ldots, k_{r}\right)$ is an ordered partition of $\ell$, i.e. an $r$-tuple that sums up to $\ell$. Using this, and the explicit expression for the inner products $\mu_{i} \cdot \mu_{j}$ (see, for example, [24]), it is easy to show that the second term in the exponential of (4.46) gives a factor

$$
\exp \left[\frac{\pi i}{N(k+N)} \sum_{\alpha} n_{\alpha} m_{\alpha} \ell_{\alpha}^{2}\right]
$$

while the first term gives

$$
\exp \left[\frac{2 \pi i}{N(k+N)} \sum_{\alpha<\beta} n_{\alpha} m_{\beta} \ell_{\alpha} \ell_{\beta}\right]
$$

None of these factors can be written in terms of $t^{ \pm 1 / 2}$ and $\lambda^{ \pm 1 / 2}$, since they involve a $1 / N$ factor in the denominator of the exponent. As explained in [19, the writhe of each of the components of the link constructed in (4.46) is $w\left(\mathcal{K}_{\alpha}\right)=n_{\alpha} m_{\alpha}$. Using (4.37) (4.38) it is easy to see that the factor (4.50) will be canceled by the correction that is needed in order to enforce the standard framing. On the other hand, the remaining factor (4.51) is due to the linking of the different components, since $\operatorname{lk}\left(K_{\alpha}, K_{\beta}\right)=-n_{\alpha} m_{\beta}, \alpha<\beta$, in this case [19]. We see that the correction factor in (4.40) is the minimal one that has to be 
introduced in order to cancel (4.51). Since the character involved in (4.48) is a rational function of $t^{ \pm 1 / 2}$ and $\lambda^{ \pm 1 / 2}$, it follows that the invariant defined in (4.40) is also a rational function of these two variables, at least in the particular case of links made up of torus knots by the above construction.

A particularly interesting example occurs when all the components of the link are the same torus knot. In this case, the resulting link is called a torus link. A torus link of $L$ components is made of $L$ torus knots of type $(n, m)$, and will be labeled by $(n L, m L)$, where $n$ and $m$ are relatively prime. For example, the Hopf link is the $(2,2)$ torus link. For torus links, the expression (4.46) can be simplified very much. It is easy to see that when $\left(n_{1}, m_{1}\right)=\cdots=\left(n_{L}, m_{L}\right)=(n, m)$, the action of the string of operators on the vacuum is exactly that of a single knot operator $(n, m)$ but in the tensor product representation $\otimes_{\alpha=1}^{L} R_{\alpha}$. This means that the vev can be computed by decomposing the tensor product and using the results of [3], without further ado. The decomposition in irreducible representations can be written as follows,

$$
\otimes_{\alpha=1}^{L} R_{\alpha}=\sum_{s} \mathcal{N}_{\Lambda_{1}, \cdots, \Lambda_{L}}^{\Lambda_{s}} R_{s}
$$

where the integers $\mathcal{N}_{\Lambda_{1}, \cdots, \Lambda_{L}}^{\Lambda_{s}}$ can be easily computed by using repeatedly the LittlewoodRichardson rule. After taking into account the correction factors (4.33), one finally obtains the following expression for the vev:

$$
\left\langle\prod_{\alpha=1}^{L} W_{\Lambda_{\alpha}}^{(n, m)}\right\rangle=\sum_{s} \mathcal{N}_{\Lambda_{1}, \cdots, \Lambda_{L}}^{\Lambda_{s}} t^{\frac{m n}{2}\left(\sum_{\alpha=1}^{L} \kappa_{\Lambda_{\alpha}}-\kappa_{\Lambda_{s}}\right)}\left\langle W_{\Lambda_{s}}^{(n, m)}\right\rangle,
$$

where the detailed expression for $\left\langle W_{\Lambda_{s}}^{(n, m)}\right\rangle$ was given in [3], and $\kappa_{\Lambda}$ has been defined in (4.36). We will use (4.53) to give some explicit results on the link invariants in section 7 .

\subsection{Some predictions for the HOMFLY polynomial of links}

The result (4.18) gives a powerful structure theorem about the link invariants derived from Chern-Simons theory. Even in the case of links in the fundamental representation (i.e. the HOMFLY polynomial) one can obtain some highly non-trivial results. To extract the consequences of the above result, it is convenient to introduce some notation. Let's consider a link $\mathcal{L}$ of $L$ components $\mathcal{K}_{\alpha}, \alpha=1, \cdots, L$. The usual HOMFLY polynomial of 
this link will be denoted by $P_{\mathcal{L}}(q, \lambda)$, and it is related to the corrected Chern-Simons vev $\langle W(\mathcal{L})\rangle$ as follows:

$$
\langle W(\mathcal{L})\rangle=\lambda^{\operatorname{lk}(\mathcal{L})}\left(\frac{\lambda^{\frac{1}{2}}-\lambda^{-\frac{1}{2}}}{q^{\frac{1}{2}}-q^{-\frac{1}{2}}}\right) P_{\mathcal{L}}(q, \lambda) .
$$

The structure theorem (4.18) says that

$$
\langle W(\mathcal{L})\rangle^{(c)}=\left(q^{-\frac{1}{2}}-q^{\frac{1}{2}}\right)^{L-2} \sum_{Q} \sum_{g \geq 0} \widehat{N}_{(\square, \cdots, \square), g, Q} \lambda^{Q}\left(q^{-\frac{1}{2}}-q^{\frac{1}{2}}\right)^{2 g} .
$$

We will first consider the simple case of a link of two components. Using (4.9), we find that the HOMFLY polynomial of the link has the following structure:

$$
P_{\mathcal{L}}(t, \lambda)=\sum_{g \geq 0} p_{2 g-1}^{\mathcal{L}}(\lambda)\left(q^{\frac{1}{2}}-q^{-\frac{1}{2}}\right)^{2 g-1}
$$

i.e. the lowest power of $q^{\frac{1}{2}}-q^{-\frac{1}{2}}$ is -1 , and the powers are congruent to $-1 \bmod 2$. Moreover, if we denote the HOMFLY polynomial of the component knots by

$$
P_{\mathcal{K}_{\alpha}}(\lambda, q)=\sum_{g \geq 0} p_{2 g}^{\mathcal{K}_{\alpha}}(\lambda)\left(q^{\frac{1}{2}}-q^{-\frac{1}{2}}\right)^{2 g}
$$

for $\alpha=1,2$, we find

$$
p_{-1}^{\mathcal{L}}(\lambda)=\lambda^{-\mathrm{k}(\mathcal{L})}\left(\lambda^{\frac{1}{2}}-\lambda^{-\frac{1}{2}}\right) p_{0}^{\mathcal{K}_{1}}(\lambda) p_{0}^{\mathcal{K}_{2}}(\lambda)
$$

The last equation comes from the requirement that there are no powers of $\left(q^{\frac{1}{2}}-q^{-\frac{1}{2}}\right)^{-2}$ in $\langle W(\mathcal{L})\rangle^{(c)}$. The results (4.56) and (4.58) capture completely the algebraic structure of the HOMFLY polynomial of a two-component link, and reproduce the results of Lickorish and Millett [15].

We can generalize the above results for links with an arbitrary number of components $L$. We introduce again some notation. $\mathcal{L}_{\alpha_{1}, \cdots, \alpha_{s}}$ will denote the sublink of $s$ components obtained from the link $\mathcal{L}$ by "forgetting" $L-s$ components, and $\left\{\alpha_{1}, \cdots, \alpha_{s}\right\} \subset\{1, \cdots, L\}$. The connected vev $\langle W(\mathcal{L})\rangle^{(c)}$ is then given by the original vev together with some corrections involving products of vevs for sublinks:

$$
\langle W(\mathcal{L})\rangle^{(c)}=\langle W(\mathcal{L})\rangle-\sum_{\alpha_{L}=1}^{L}\left\langle W\left(\mathcal{L}_{\alpha_{1}, \cdots, \alpha_{L-1}}\right)\right\rangle\left\langle W\left(\mathcal{L}_{\alpha_{L}}\right)\right\rangle+\cdots
$$


By induction on the number of components, and using (4.59)(4.55), it is very easy to prove that the HOMFLY polynomial of the link has the following structure:

$$
P_{\mathcal{L}}(q, \lambda)=\sum_{g \geq 0} p_{2 g+1-L}^{\mathcal{L}}(\lambda)\left(q^{\frac{1}{2}}-q^{-\frac{1}{2}}\right)^{2 g+1-L},
$$

i.e. the lowest power of $q^{\frac{1}{2}}-q^{-\frac{1}{2}}$ is $1-L$. This has been also proved in [15]. Due to our correction factor (4.34), it is convenient to introduce the following polynomials in $\lambda$ :

$$
\widetilde{p}_{k}^{\mathcal{L}_{\alpha_{1}}, \cdots, \alpha_{s}}(\lambda)=\lambda^{\mathrm{lk}\left(\mathcal{L}_{\alpha_{1}, \cdots, \alpha_{s}}\right)} p_{k}^{\mathcal{L}_{\alpha_{1}, \cdots, \alpha_{s}}}(\lambda) .
$$

Finally, we will write

$$
\langle W(\mathcal{L})\rangle^{(c)}=\left(\frac{\lambda^{\frac{1}{2}}-\lambda^{-\frac{1}{2}}}{q^{\frac{1}{2}}-q^{-\frac{1}{2}}}\right) \sum_{g \geq 0} \widetilde{p}_{2 g+1-L}^{(c), \mathcal{L}}(\lambda)\left(q^{\frac{1}{2}}-q^{-\frac{1}{2}}\right)^{2 g+1-L} .
$$

The structure theorem (3.31) then states that

$$
\widetilde{p}_{1-L}^{(c), \mathcal{L}}(\lambda)=\widetilde{p}_{3-L}^{(c), \mathcal{L}}(\lambda)=\cdots=\widetilde{p}_{L-3}^{(c), \mathcal{L}}(\lambda)=0 .
$$

This implies that the polynomials $p_{k}^{\mathcal{L}}(\lambda)$ of the HOMFLY polynomial of a link, for $k=$ $1-L, 3-L, \cdots, L-3$, are completely determined by the HOMFLY polynomial of its sublinks. As a first consequence, we find that $\widetilde{p}_{1-L}^{(c), \mathcal{L}}(\lambda)=0$ gives the generalization of (4.58) to an arbitrary link:

$$
p_{1-L}^{\mathcal{L}}(\lambda)=\lambda^{-\operatorname{lk}(\mathcal{L})}\left(\lambda^{\frac{1}{2}}-\lambda^{-\frac{1}{2}}\right)^{L-1} \prod_{\alpha=1}^{L} p_{0}^{\mathcal{K}_{\alpha}}(\lambda) .
$$

This is easy to prove by induction on the number of components: since $\widetilde{p}_{1-L}^{(c), \mathcal{L}}(\lambda)=0$, one can extract the coefficient of the lowest power of $q^{\frac{1}{2}}-q^{-\frac{1}{2}}$ in $\langle W(\mathcal{L})\rangle$ from the terms involving products of vevs of sublinks in the expansion of the connected piece. One sees immediately that the relevant part of these vevs is again the coefficient of the lowest power of $q^{\frac{1}{2}}-q^{-\frac{1}{2}}$. But because of the induction hypothesis, these in turn can be evaluated by factorization into their knots. This means that the coefficient $\widetilde{p}_{1-L}^{\mathcal{L}}$ can be evaluated from $\prod_{\alpha=1}^{L}\left\langle W\left(\mathcal{K}_{\alpha}\right)\right\rangle$, and this proves (4.64). This formula was in fact obtained by Lickorish and Millett in 15 using the skein relation. In our context, this is just the simplest prediction of (4.63), which gives much more relations. For example, for links with $L=3$, the equality $\widetilde{p}_{0}^{(c), \mathcal{L}}(\lambda)=0$ implies that

$$
\begin{aligned}
\widetilde{p}_{0}^{\mathcal{L}}(\lambda) & =\left(\lambda^{\frac{1}{2}}-\lambda^{-\frac{1}{2}}\right)\left(p_{0}^{\mathcal{K}_{1}}(\lambda) \tilde{p}_{1}^{\mathcal{L}_{23}}(\lambda)+\text { perms }\right) \\
& -2\left(\lambda^{\frac{1}{2}}-\lambda^{-\frac{1}{2}}\right)^{2}\left(p_{2}^{\mathcal{K}_{1}}(\lambda) p_{0}^{\mathcal{K}_{2}}(\lambda) p_{0}^{\mathcal{K}_{3}}(\lambda)+\text { perms }\right) .
\end{aligned}
$$

For links with more components, one obtains more complicated equations which can be summarized as in (4.63) and give new results on the algebraic structure of the HOMFLY polynomial of links. 


\section{Lagrangian submanifold for torus links}

As noted before, in order to describe the large $N$ closed string dual for knot and link invariants, we need to construct a suitable Lagrangian submanifold on the blown up conifold geometry which corresponds to the D-branes after the conifold transition. This has been done for the case of the trivial knot in [2]. In this section we wish to generalize this construction for certain links known as algebraic links (see for example [25] for a discussion of algebraic links). These are links that can be obtained by intersecting a plane curve in $\mathbf{C}^{2}$

$$
F\left(\zeta_{1}, \zeta_{2}\right)=0
$$

with a three-sphere $\left|\zeta_{1}\right|^{2}+\left|\zeta_{2}\right|^{2}=R$. Torus links are in fact algebraic, since a torus link of type $(n, m)$ is described by the equation

$$
\zeta_{1}^{n}+\zeta_{2}^{m}=0
$$

This can be easily seen by writing $\zeta_{1}=r_{1} \mathrm{e}^{i \theta_{1}}, \zeta_{2}=r_{2} \mathrm{e}^{i \theta_{2}}$. The number of components of this link is precisely $L=\operatorname{gcd}(n, m)$. It is possible to obtain a more general algebraic equation describing the same torus link by taking the right hand side of (5.2) to be a polynomial in $\zeta_{1}, \zeta_{2}$ of lower degree (provided the radius $R$ of the three-sphere is big enough). It is important to notice that not all links and knots are algebraic.

The equation (5.1) describes an algebraic curve in $\mathbf{C}^{2}$. However on the blown up conifold geometry we are interested in a 3 dimensional Lagrangian submanifold. How does one get a canonical such manifold from the above construction? There are two things that need to be changed here: first, we need a Lagrangian submanifold rather than an algebraic one. Secondly we need a 3 dimensional manifold and not a 2-dimensional one.

To remedy the first difficulty, notice that one can perform a hyperKähler rotation and obtain a two-dimensional (real) submanifold which is Lagrangian for the canonical Kähler form $\omega=(i / 2) \sum_{k=1}^{2} d \zeta_{k} \wedge d \bar{\zeta}_{k}$. The resulting equation is simply,

$$
F\left(\mathrm{e}^{i \theta / 2} \zeta_{1}-\mathrm{e}^{-i \theta / 2} \bar{\zeta}_{2}, \mathrm{e}^{i \theta / 2} \zeta_{2}+\mathrm{e}^{-i \theta / 2} \bar{\zeta}_{1}\right)=0
$$

whee $\theta$ is a real parameter. To prove that this submanifold is Lagrangian, one considers the equation $d F=0$ and its complex conjugate, which read:

$$
\begin{aligned}
& \left(\partial_{1} F\right)\left(\mathrm{e}^{i \theta / 2} d \zeta_{1}-\mathrm{e}^{-i \theta / 2} d \bar{\zeta}_{2}\right)=-\left(\partial_{2} F\right)\left(\mathrm{e}^{i \theta / 2} d \zeta_{2}+\mathrm{e}^{-i \theta / 2} d \bar{\zeta}_{1}\right) \\
& \left(\overline{\partial_{1} F}\right)\left(\mathrm{e}^{-i \theta / 2} d \bar{\zeta}_{1}-\mathrm{e}^{i \theta / 2} d \zeta_{2}\right)=-\left(\overline{\partial_{2} F}\right)\left(\mathrm{e}^{-i \theta / 2} d \bar{\zeta}_{2}+\mathrm{e}^{i \theta / 2} d \zeta_{1}\right)
\end{aligned}
$$


Assuming that $\partial_{1} F \neq 0$, and wedging the first equation with $\mathrm{e}^{i \theta / 2} d \zeta_{2}+\mathrm{e}^{-i \theta / 2} d \bar{\zeta}_{1}$ and the second equation with $\mathrm{e}^{-i \theta / 2} d \bar{\zeta}_{2}+\mathrm{e}^{i \theta / 2} d \zeta_{1}$, we easily obtain that $\left.\omega\right|_{F=0}=0$, for any $\theta$.

To remedy the second difficulty we recall that we are considering a Lagrangian submanifold in $\mathcal{O}(-1)+\mathcal{O}(-1) \rightarrow \mathbf{P}^{1}$. For each point of $\mathbf{P}^{1}$ we have a copy of $\mathbf{C}^{2}$ and the $\mathbf{S}^{3}$ should be identified with the large $\mathbf{S}^{3}$ sitting in $\mathbf{C}^{2}$. The condition that the Lagrangian submanifold after transition corresponds to a specific link before transition is simply that the Lagrangian submanifold intersects the $\mathbf{S}^{3}$ at infinity along the corresponding link. This is precisely the case for the above construction. Thus the above two dimensional construction can be viewed in principle as a 2 dimensional slice of a 3 d Lagrangian submanifold, where one dimension of the Lagrangian submanifold projects to a curve in $\mathbf{P}^{1}$.

In order to complete the story, we need to construct that third direction of the Lagrangian submanifold. The idea is to use the $S O(2)$ rotation symmetry of $\mathcal{O}(-1)+$ $\mathcal{O}(-1) \rightarrow \mathbf{P}^{1}$ (where one thinks of the fiber directions as two copies of spinors and $S O(2)$ acts on $\mathbf{P}^{1}$ by the standard rotation), and rotate the 2-dimensional Lagrangian submanifold to get a 3-dimensional Lagrangian submanifold. In particular consider the 3-dimensional manifold which is given by the above Lagrangian submanifold, where one interprets $\theta$ as the angle along the equator of $\mathbf{P}^{1}$. In other words, the projection of the 3 -manifold over $\mathbf{P}^{1}$ is given by the equator parameterized by $\theta$ and over each point the fiber is the 2 dimensional Lagrangian submanifold constructed above. Note that the $\theta$ dependence in the above fibration is consistent with the $S O(2)$ action on the fibers (being spinors over the sphere). This manifold makes sense if we get the same fiber over $\theta=0$ and $\theta=2 \pi$, which means that this construction only makes sense for $F\left(\zeta_{1}, \zeta_{2}\right)= \pm F\left(-\zeta_{1},-\zeta_{2}\right)$. We will now show that this 3 dimensional submanifold is indeed Lagrangian, at least for a specific choice of metric on $\mathcal{O}(-1)+\mathcal{O}(-1) \rightarrow \mathbf{P}^{1}$ which is symplectically equivalent to that of the Ricci-flat metric.

The Ricci-flat metric on this Calabi-Yau has a Kähler form that can be written as

$$
\omega=\frac{i}{2} \partial \bar{\partial} K(U)+\omega_{\mathbf{P}^{1}} .
$$

In this equation, $\omega_{\mathbf{P}^{1}}$ is the usual symmetric Kähler form on the sphere and $K(U)$ is a Kähler potential depending on the variable

$$
U=\left(1+|z|^{2}\right)\left(\left|\zeta_{1}\right|^{2}+\left|\zeta_{2}\right|^{2}\right),
$$

where $z$ is a complex coordinate on $\mathbf{P}^{1}$. Note that $U$ is simply the norm of the spinor bundles with respect to the constant curvature metric on $\mathbf{P}^{1}$. Since we are only interested 
in the topological string amplitudes only the Kähler class of the metric should be relevant and not whether it is Ricci-flat or not. For this any $K(U)$ will be sufficient. We take $K(U)=U$ (which is not a Ricci-flat metric). In this case the Kähler form is given by

$$
\frac{i}{2} \sum_{k=1}^{2}\left(\left(1+|z|^{2}\right)\left(d \zeta_{k} \wedge d \bar{\zeta}_{k}\right)+\bar{z} \zeta_{k} d z \wedge d \bar{\zeta}_{k}+z \bar{\zeta}_{k} d \zeta_{k} \wedge d \bar{z}\right)+\mathcal{O}(d z \wedge d \bar{z})
$$

where the terms involving $d z \wedge d \bar{z}$ are clearly vanishing over the 3 dimensional manifold we have constructed. Using this explicit expression for the Kähler form, it is easy to prove that for $z=\mathrm{e}^{i \theta}$ (i.e. the equator of the sphere) the equation (5.3) describes a Lagrangian submanifold which has the topology of a surface bundle over the equator. We have then obtained a Lagrangian submanifold in the resolved conifold corresponding to the algebraic link described by (5.1).

Notice that as explained above the equation (5.1) has monodromy as we go around the equator, therefore it is not always well-defined for arbitrary torus links. For (5.2), we must have $(n, m)$ both even or both odd. If this is the case, it is natural to propose that (5.3) in fact describes the Lagrangian submanifold corresponding to the algebraic link (5.1) after the transition. As an evidence for the above proposal, note that this construction for the case of $(n, m)=(1,1)$ which corresponds to the unknot reproduces the result of [2]. Also for the case of $(n, m)=(2,2)$, which corresponds to the Hopf link (and similarly

for $(n, m)=(k, k))$, the above construction agrees with a simple generalization of the construction in [2] to this case. This provides further evidence for our construction.

\section{Explicit results and examples}

In this section we illustrate the results of the previous sections with explicit computations in Chern-Simons. The equations (3.19) and (4.18) give important and highly nontrivial structural predictions for the link invariants of Chern-Simons theory. The fact that they turn out to be true in all the cases that we have checked gives strong evidence for the D-brane degeneracy picture advocated in this paper. The results presented below are just an illustrative sample of all the examples that we have computed, involving many different torus knots and links. Since the resulting expressions are typically complicated, we have presented examples involving representations with a small number of boxes and knots and links with only a few crossings. Further examples are easily obtained with our results for torus links. 
In the first subsection we present a computation of the new integer invariants $\widehat{N}_{R, g, Q}$ in the case of knots. The computation of the generating functions $f_{R}$ has been done in [3] for torus knots and in [4] for some other knots with up to nine crossings. From these generating functions, and using (3.21) and the explicit expression of the matrix $M_{R R^{\prime}}(q)$, we can extract the new integer invariants introduced in section 3 . The fact that the functions $\widehat{f}_{R}$ turn out to have the simple structure predicted in (3.20) is highly nontrivial from the point of view of Chern-Simons, since the entries of the inverse matrix of $M_{R R^{\prime}}(q)$ are rational functions with very complicated denominators. The integrality property of the invariants $\widehat{N}_{R, g, Q}$ is clearly stronger than the integrality property of $N_{R, Q, s}$, and gives a powerful check of the arguments presented in section 3

After presenting the results for knots, we consider the case of links and we illustrate the arguments presented in section 4. For links, the structural results that we have obtained are already very strong when all the components are in the fundamental representation, as we have seen in subsection 4.6. Our results also confirm that the proposal (4.40) is the right prescription to obtain results compatible with the dual description in terms of topological strings.

In principle, the integer invariants $\widehat{N}_{R, g, Q}$ can be computed on the gravity side, after the transition, by looking at the moduli space of Riemann surfaces with boundaries on the Lagrangian submanifold. It would be very interesting to compute some of these numbers by using the explicit construction for torus links presented in section 5 .

\subsection{Knots}

We will first give some examples of the integer invariants $\widehat{N}_{R, g, Q}$ for the trefoil knot, for representations of up to 3 boxes. The values of the invariants can be read from equations (4.7), (4.13) and (4.21) of [3], and are presented in tables 1-6.

\begin{tabular}{|lrrr|}
\hline$g$ & $Q=1 / 2$ & $3 / 2$ & $5 / 2$ \\
\hline 0 & 2 & -3 & 1 \\
1 & 1 & -1 & 0 \\
\hline
\end{tabular}

Table 1: The integers $\widehat{N}_{\square, g, Q}$ for the trefoil knot. 


\begin{tabular}{|lrrrrr|}
\hline$g$ & $Q=1$ & 2 & 3 & 4 & 5 \\
\hline 0 & 2 & -8 & 12 & -8 & 2 \\
1 & 1 & -6 & 10 & -6 & 1 \\
2 & 0 & -1 & 2 & -1 & 0 \\
\hline
\end{tabular}

Table 2: The integers $\widehat{N}_{\square, g, Q}$ for the trefoil knot.

\begin{tabular}{|lrrrrr|}
\hline$g$ & $Q=1$ & 2 & 3 & 4 & 5 \\
\hline 0 & 4 & -16 & 24 & -16 & 4 \\
1 & 4 & -20 & 32 & -20 & 4 \\
2 & 1 & -8 & 14 & -8 & 1 \\
3 & 0 & -1 & 2 & -1 & 0 \\
\hline
\end{tabular}

Table 3: The integers $\widehat{N}_{日, g, Q}$ for the trefoil knot.

\begin{tabular}{|lrrrrrrr|}
\hline$g$ & $Q=3 / 2$ & $5 / 2$ & $7 / 2$ & $9 / 2$ & $11 / 2$ & $13 / 2$ & $15 / 2$ \\
\hline 0 & 2 & -18 & 64 & -116 & 114 & -58 & 12 \\
1 & 1 & -21 & 106 & -232 & 251 & -131 & 26 \\
2 & 0 & -8 & 67 & -187 & 227 & -121 & 22 \\
3 & 0 & -1 & 19 & -74 & 103 & -55 & 8 \\
4 & 0 & 0 & 2 & -14 & 23 & -12 & 1 \\
5 & 0 & 0 & 0 & -1 & 2 & -1 & 0 \\
\hline
\end{tabular}

Table 4: The integers $\widehat{N}_{\square, g, Q}$ for the trefoil knot.

\begin{tabular}{|lrrrrrrr|}
\hline$g$ & $Q=3 / 2$ & $5 / 2$ & $7 / 2$ & $9 / 2$ & $11 / 2$ & $13 / 2$ & $15 / 2$ \\
\hline 0 & 11 & -99 & 332 & -558 & 507 & -239 & 46 \\
1 & 15 & -201 & 842 & -1627 & 1612 & -796 & 155 \\
2 & 7 & -164 & 910 & -2080 & 2275 & -1172 & 224 \\
3 & 1 & -66 & 528 & -1475 & 1792 & -947 & 167 \\
4 & 0 & -13 & 171 & -614 & 833 & -443 & 66 \\
5 & 0 & -1 & 29 & -148 & 226 & -119 & 13 \\
6 & 0 & 0 & 2 & -19 & 33 & -17 & 1 \\
7 & 0 & 0 & 0 & -1 & 2 & -1 & 0 \\
\hline
\end{tabular}

Table 5: The integers $\widehat{N}_{\boxplus, g, Q}$ for the trefoil knot. 


\begin{tabular}{|lrrrrrrr|}
\hline$g$ & $Q=3 / 2$ & $5 / 2$ & $7 / 2$ & $9 / 2$ & $11 / 2$ & $13 / 2$ & $15 / 2$ \\
\hline 0 & 12 & -108 & 352 & -568 & 492 & -220 & 40 \\
1 & 26 & -306 & 1180 & -2136 & 2006 & -950 & 180 \\
2 & 22 & -366 & 1740 & -3618 & 3728 & -1864 & 358 \\
3 & 8 & -230 & 1431 & -3504 & 3978 & -2066 & 383 \\
4 & 1 & -79 & 698 & -2077 & 2603 & -1378 & 232 \\
5 & 0 & -14 & 200 & -761 & 1057 & -561 & 79 \\
6 & 0 & -1 & 31 & -167 & 259 & -136 & 14 \\
7 & 0 & 0 & 2 & -20 & 35 & -18 & 1 \\
8 & 0 & 0 & 0 & -1 & 2 & -1 & 0 \\
\hline
\end{tabular}

Table 6: The integers $\widehat{N}_{\text {日,g,Q }}$ for the trefoil knot.

In addition, we present also the the values of the integers $\widehat{N}_{\square, g, Q}, \widehat{N}_{\square, g, Q}$ and $\widehat{N}_{\boxminus, g, Q}$ for the knots $4_{1}, 5_{1}$ and $6_{1}$ shown in figure 1 . The results are collected in tables 7-15. Knots $4_{1}$, and $6_{1}$ are not torus knots. The values of the integer invariants have been obtained from the expressions for $f_{\square}, f_{\square}$ and $f_{日}$ presented in $\llbracket$. The knot $5_{1}$ is a torus knot and the integer invariants have been obtained after using the general formula for this type of knots provided in [3]. The following tables contain the values that take all these integer invariants. Notice that for the amphicheiral knot $4_{1}$ the results are consistent with the fact that for this type of knots $f_{R}(q, \lambda)$ are invariant under $q \rightarrow q^{-1}, \lambda \rightarrow \lambda^{-1}$. Using (3.12) and (3.19) it is easy to prove that amphicheiral knots satisfy

$$
\widehat{N}_{R, g, Q}=(-1)^{\ell} \widehat{N}_{R^{t}, g,-Q}
$$

as one can see for $R=\square$, $\square$ and $\theta$ in tables 7-9.

\begin{tabular}{|lrrrr|}
\hline$g$ & $Q=-3 / 2$ & $-1 / 2$ & $1 / 2$ & $3 / 2$ \\
\hline 0 & 1 & -2 & 2 & -1 \\
0 & 0 & -1 & 1 & 0 \\
\hline
\end{tabular}

Table 7: The integers $\widehat{N}_{\square, g, Q}$ for the figure-eight knot $4_{1}$.

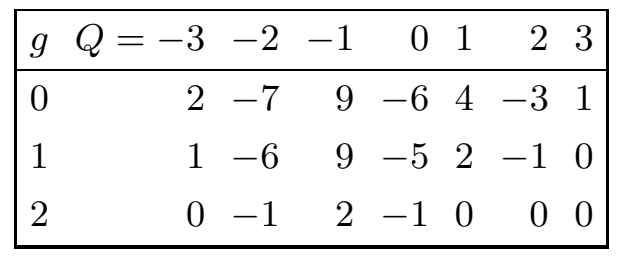

Table 8: The integers $\widehat{N}_{\square, g, Q}$ for the figure-eight knot $4_{1}$. 


\begin{tabular}{|lrrrrrrr|}
\hline$g$ & $Q=-3$ & -2 & -1 & 0 & 1 & 2 & 3 \\
\hline 0 & 1 & -3 & 4 & -6 & 9 & -7 & 2 \\
1 & 0 & -1 & 2 & -5 & 9 & -6 & 1 \\
2 & 0 & 0 & 0 & -1 & 2 & -1 & 0 \\
\hline
\end{tabular}

Table 9: The integers $\widehat{N}_{\mathrm{B}, g, Q}$ for the figure-eight knot $4_{1}$.

\begin{tabular}{|lrrr|}
\hline$g$ & $Q=3 / 2$ & $5 / 2$ & $7 / 2$ \\
\hline 0 & 3 & -5 & 2 \\
1 & 4 & -5 & 1 \\
2 & 1 & -1 & 0 \\
\hline
\end{tabular}

Table 10: The integers $\widehat{N}_{\square, g, Q}$ for the knot $5_{1}$.

\begin{tabular}{|rrrrrr|}
\hline$g$ & $Q=3$ & 4 & 5 & 6 & 7 \\
\hline 0 & 20 & -80 & 120 & -80 & 20 \\
1 & 60 & -260 & 400 & -260 & 60 \\
2 & 69 & -336 & 534 & -336 & 69 \\
3 & 38 & -221 & 366 & -221 & 38 \\
4 & 10 & -78 & 136 & -78 & 10 \\
5 & 1 & -14 & 26 & -14 & 1 \\
6 & 0 & -1 & 2 & -1 & 0 \\
\hline
\end{tabular}

Table 11: The integers $\widehat{N}_{\square, g, Q}$ for the knot $5_{1}$.

\begin{tabular}{|rrrrrr|}
\hline$g$ & $Q=3$ & 4 & 5 & 6 & 7 \\
\hline 0 & 30 & -120 & 180 & -120 & 30 \\
1 & 115 & -490 & 750 & -490 & 115 \\
2 & 176 & -819 & 1286 & -819 & 176 \\
3 & 137 & -724 & 1174 & -724 & 137 \\
4 & 57 & -365 & 616 & -365 & 57 \\
5 & 12 & -105 & 186 & -105 & 12 \\
6 & 1 & -16 & 30 & -16 & 1 \\
7 & 0 & -1 & 2 & -1 & 0 \\
\hline
\end{tabular}

Table 12: The integers $\widehat{N}_{\text {日, }, Q, Q}$ for the knot $5_{1}$. 


\begin{tabular}{|lrrrrr|}
\hline$g$ & $Q=-3 / 2$ & $-1 / 2$ & $1 / 2$ & $3 / 2$ & $5 / 2$ \\
\hline 0 & 1 & -1 & -1 & 2 & -1 \\
1 & 0 & -1 & 0 & 1 & 0 \\
\hline
\end{tabular}

Table 13: The integers $\widehat{N}_{\square, g, Q}$ for the knot $6_{1}$.

\begin{tabular}{|lrrrrrrrrr|}
\hline$g$ & $Q=-3$ & -2 & -1 & 0 & 1 & 2 & 3 & 4 & 5 \\
\hline 0 & 2 & -4 & -1 & 7 & -4 & -6 & 15 & -13 & 4 \\
1 & 1 & -5 & 2 & 6 & -4 & -10 & 25 & -19 & 4 \\
2 & 0 & -1 & 1 & 1 & -1 & -6 & 13 & -8 & 1 \\
3 & 0 & 0 & 0 & 0 & 0 & -1 & 2 & -1 & 0 \\
\hline
\end{tabular}

Table 14: The integers $\widehat{N}_{\square, g, Q}$ for the knot $6_{1}$.

\begin{tabular}{|rrrrrrrrrr|}
\hline$g$ & $Q=-3$ & -2 & -1 & 0 & 1 & 2 & 3 & 4 & 5 \\
\hline 0 & 1 & -2 & 0 & 3 & -4 & -12 & 26 & -21 & 6 \\
1 & 0 & -1 & 1 & 1 & 0 & -26 & 60 & -46 & 11 \\
2 & 0 & 0 & 0 & 0 & 0 & -22 & 50 & -34 & 6 \\
3 & 0 & 0 & 0 & 0 & 0 & -8 & 17 & -10 & 1 \\
4 & 0 & 0 & 0 & 0 & 0 & -1 & 2 & -1 & 0 \\
\hline
\end{tabular}

Table 15: The integers $\widehat{N}_{\text {日, }, Q}$ for the knot $6_{1}$. 


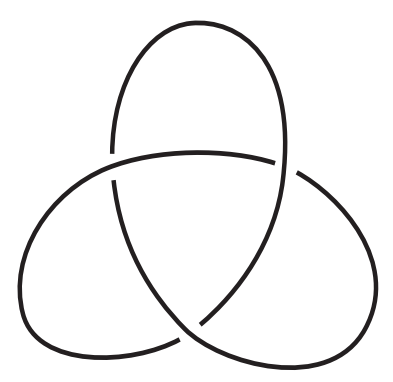

$3_{1}$

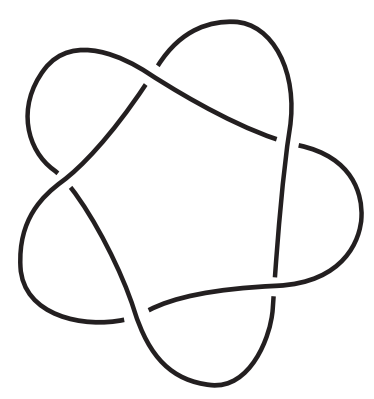

$5_{1}$

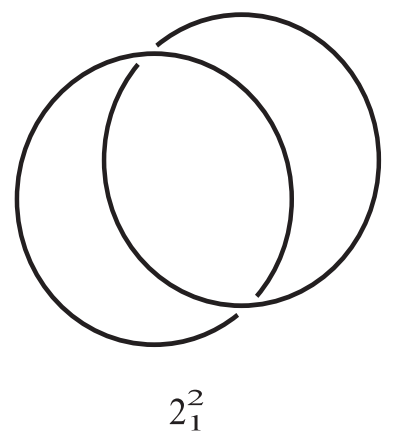

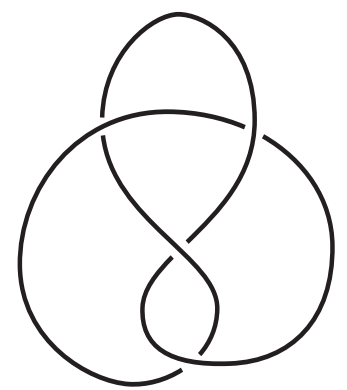

$4_{1}$

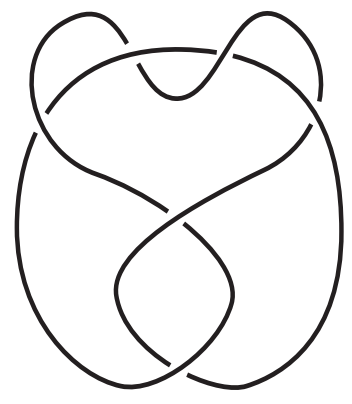

$6_{1}$

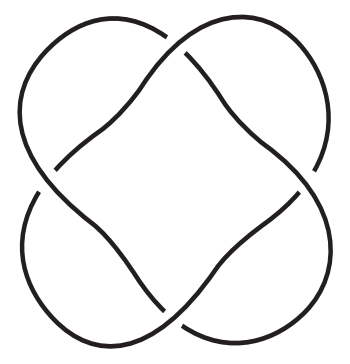

$4_{1}^{2}$

Fig. 1: Some of the knots and links considered in the paper. The trefoil knot $3_{1}$ and the knot $5_{1}$ are the torus knots $(3,2)$ and $(5,2)$, in the notation used in subsection 4.5. The figure-eight knot $4_{1}$ and the knot $6_{1}$ are not torus knots. The first one is amphicheiral. The two-component links $2_{1}^{2}$ and $4_{1}^{2}$ correspond to the torus links $(2,2)$ and $(2,4)$ in the notation used in subsection 4.5 .

\subsection{Links}

We now give explicit results for some torus links. The simplest link is the Hopf link, which is the $(2,2)$ torus link. It has two components which are both the unknot. Before presenting the results, it is worthwhile to show that the correction factor in (4.34) is crucial 
to agree with the string predictions. The unnormalized HOMFLY polynomial of the Hopf link is given by:

$$
\left(\frac{\lambda^{\frac{1}{2}}-\lambda^{-\frac{1}{2}}}{q^{\frac{1}{2}}-q^{-\frac{1}{2}}}\right)\left(\frac{\lambda^{\frac{3}{2}}-\lambda^{\frac{1}{2}}}{q^{\frac{1}{2}}-q^{-\frac{1}{2}}}-\lambda^{\frac{1}{2}}\left(q^{\frac{1}{2}}-q^{-\frac{1}{2}}\right)\right)
$$

The linking number of the two unknots is -1 , and according to (4.34) the vev $\left\langle\operatorname{Tr} U_{1} \operatorname{Tr} U_{2}\right\rangle$ should be equal to the unnormalized HOMFLY polynomial, times $\lambda^{-1}$. Without this factor, the connected vev (4.9) does not have the structure predicted by (4.6). Once the right prescription has been used, one obtains:

$$
f_{(\square, \square)}(q, \lambda)=-\lambda^{-1}(\lambda-1)
$$

Using all our previous results, we can compute the $f$ 's up to order $\ell_{1}+\ell_{2}=4$. At order three, we find:

$$
\begin{aligned}
& f_{(\square, \square)}(q, \lambda)=-\lambda^{-1}\left(\lambda^{\frac{1}{2}}-\lambda^{-\frac{1}{2}}\right) q^{-\frac{1}{2}}, \\
& f_{(\text {日, } \square)}(q, \lambda)=\lambda^{-1}\left(\lambda^{\frac{1}{2}}-\lambda^{-\frac{1}{2}}\right) q^{\frac{1}{2}},
\end{aligned}
$$

while at order four we get:

$$
\begin{aligned}
& f_{\text {(س, 口) }}(q, \lambda)=-\lambda^{-2}(\lambda-1) q^{-1}, \\
& f_{(\boxminus, \square)}(q, \lambda)=\lambda^{-2}(\lambda-1), \\
& f_{\text {(日, } \square)}(q, \lambda)=-\lambda^{-2}(\lambda-1) q \text {, } \\
& f_{\text {(四, 口) }}(q, \lambda)=\lambda^{-2}(\lambda-1) q^{-2}\left(\lambda q+q^{2}-q-1\right) \text {, }
\end{aligned}
$$

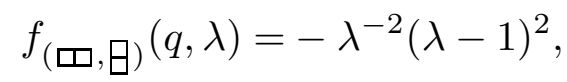

$$
\begin{aligned}
& f_{\text {(日, 日) }}(q, \lambda)=\lambda^{-2}(\lambda-1)\left(\lambda q-q^{2}-q+1\right) \text {. }
\end{aligned}
$$

The next link in complexity is the torus link $(2,4)$, again a two-component link made of two unknots, with linking number -2 . Up to order three, the results are:

$$
\begin{aligned}
& f_{(\square, \square)}(q, \lambda)=\lambda^{-1} q^{-1}(\lambda-1)\left(\lambda q-q^{2}-q-1\right), \\
& f_{\text {(ロ, 口) }}(q, \lambda)=\lambda^{-\frac{3}{2}} q^{-\frac{5}{2}}(\lambda-1)(1+q)\left(\lambda q-q^{2}-1\right), \\
& f_{\text {(日, } \mathbf{~})}(q, \lambda)=-\lambda^{-\frac{3}{2}} q^{-\frac{1}{2}}(\lambda-1)(1+q)\left(\lambda q-q^{2}-1\right) \text {. }
\end{aligned}
$$

Finally, we give a simple example of a link of three components: the link $(3,3)$, made up of three unknots. One finds:

$$
f_{(\square, \square, \square)}(q, \lambda)=\left(q^{-\frac{1}{2}}-q^{\frac{1}{2}}\right)\left\{\lambda^{-\frac{3}{2}}\left(4+\left(q^{-\frac{1}{2}}-q^{\frac{1}{2}}\right)^{2}\right)-\lambda^{-\frac{1}{2}}\left(5+\left(q^{-\frac{1}{2}}-q^{\frac{1}{2}}\right)^{2}\right)\right\},
$$


in agreement with the structure results (3.31) (4.55).

Using now the results of section 4 , we can give the integer invariants $\widehat{N}_{\left(R_{1}, R_{2}\right), g, Q}$ for the Hopf link, for $\ell_{1}+\ell_{2} \leq 4$. For $\left(R_{1}, R_{2}\right)=(\square, \square)$, we simply find

$$
\widehat{N}_{(\square, \square), 0,0}=-\widehat{N}_{(\square, \square), 0,-1}=-1,
$$

and the rest of them are zero. For $\left(R_{1}, R_{2}\right)=(\square, \square)$, we find:

$$
\widehat{N}_{(\square, \square), 0,-1 / 2}=-\widehat{N}_{(\text {凹 }, \text { 口) }, 0,-3 / 2}=-1,
$$

and $N_{(日, \square), g, Q}=0$ for all $g, Q$. For $\left(R_{1}, R_{2}\right)=($ س, 口), we have

$$
\widehat{N}_{(\text {Ш 口) }), 0,-1}=-\widehat{N}_{(\text {凹, 口),0,-2 }}=-1,
$$

and $N_{(\Xi, \square), g, Q}=N_{(日, \square), g, Q}=0$ for all $g, Q$. For $\left(R_{1}, R_{2}\right)=($ 日, 凹), one simply has

$$
\widehat{N}_{(\text {日,四 }), 0,0}=-1,
$$

and $N_{(日, \boxminus), g, Q}=0$ for all $g, Q$. Finally, for $\left(R_{1}, R_{2}\right)=(\square, \square)$ the integer invariants are given in table 7 .

$$
\begin{array}{|lrr|}
\hline g & Q=0 & 1 \\
\hline 0 & -3 & 1 \\
1 & -1 & 0 \\
\hline
\end{array}
$$

Table 16: The integers $\widehat{N}_{(\text {四,四),g,Q}}$ for the Hopf link.

For the torus link $(2,4)$, the integer invariants up to order four are presented in tables $17-24$.

$$
\begin{array}{|lrrr|}
\hline g & Q=-1 & 0 & 1 \\
\hline 0 & 3 & -4 & 1 \\
1 & 1 & -1 & 0 \\
\hline
\end{array}
$$

Table 17: The integers $\widehat{N}_{(\square, \square), g, Q}$ for the link $(2,4)$.

$$
\begin{array}{|lrrr|}
\hline g & Q=-3 / 2 & -1 / 2 & 1 / 2 \\
\hline 0 & 6 & -9 & 3 \\
1 & 5 & -6 & 1 \\
2 & 1 & -1 & 0 \\
\hline
\end{array}
$$

Table 18: The integers $\widehat{N}_{(\text {口, }, \text { 口),g,Q }}$ for the link $(2,4)$. 


$$
\begin{array}{|lrrr|}
\hline g & Q=-3 / 2 & -1 / 2 & 1 / 2 \\
\hline 0 & 2 & -3 & 1 \\
1 & 1 & -1 & 0 \\
\hline
\end{array}
$$

Table 19: The integers $\widehat{N}_{(日, \square), g, Q}$ for the torus link $(2,4)$.

$$
\begin{array}{|lrrr|}
\hline g & Q=-2 & -1 & 0 \\
\hline 0 & 10 & -16 & 6 \\
1 & 15 & -20 & 5 \\
2 & 7 & -8 & 1 \\
3 & 1 & -1 & 0 \\
\hline
\end{array}
$$

Table 20: The integers $\widehat{N}_{(\square, \square), g, Q}$ for the torus link $(2,4)$.

\begin{tabular}{|lrrr|}
\hline$g$ & $Q=-2$ & -1 & 0 \\
\hline 0 & 5 & -8 & 3 \\
1 & 5 & -6 & 1 \\
2 & 1 & -1 & 0 \\
\hline
\end{tabular}

Table 21: The integers $\widehat{N}_{(\boxminus, \square), g, Q}$ for the torus link $(2,4)$.

The integers $\widehat{N}_{(\text {(日, 口),g,Q }}$ all vanish.

\begin{tabular}{|lrrrr|}
\hline$g$ & $Q=-2$ & -1 & 0 & 1 \\
\hline 0 & 48 & -93 & 54 & -9 \\
1 & 106 & -172 & 72 & -6 \\
2 & 99 & -137 & 39 & -1 \\
3 & 47 & -57 & 10 & 0 \\
4 & 11 & -12 & 1 & 0 \\
5 & 1 & -1 & 0 & 0 \\
\hline
\end{tabular}

Table 22: The integers $\widehat{N}_{(\square, \square), g, Q}$ for the torus link $(2,4)$. 


\begin{tabular}{|lrrrr|}
\hline$g$ & $Q=-2$ & -1 & 0 & 1 \\
\hline 0 & 26 & -47 & 24 & -3 \\
1 & 45 & -67 & 23 & -1 \\
2 & 30 & -38 & 8 & 0 \\
3 & 9 & -10 & 1 & 0 \\
4 & 1 & -1 & 0 & 0 \\
\hline
\end{tabular}

Table 23: The integers $\widehat{N}_{(\square, \Theta), g, Q}$ for the torus link $(2,4)$.

\begin{tabular}{|lrrrr|}
\hline$g$ & $Q=-2$ & -1 & 0 & 1 \\
\hline 0 & 12 & -21 & 10 & -1 \\
1 & 16 & -22 & 6 & 0 \\
2 & 7 & -8 & 1 & 0 \\
3 & 1 & -1 & 0 & 0 \\
\hline
\end{tabular}

Table 24: The integers $\widehat{N}_{(日, 日), g, Q}$ for the torus link $(2,4)$.

\section{Acknowledgments}

We would like to thank K. Hori, M. Liu, J. Maldacena, G. Moore, H. Ooguri and C. Taubes for useful conversations. M.M. would like to thank the High Energy Theory Group at Harvard for hospitality. J.M.F.L. would like to thank the Department of Physics at the University of Maryland, where part of this work was done, for hospitality. The work of J.M.F.L. is supported in part by DGICYT under grant PB96-0960. The work of M.M. is supported by DOE grant DE-FG02-96ER40959. The work of C.V. is partially supported by NSF grant PHY-98-02709. 


\section{References}

[1] R. Gopakumar and C. Vafa, "On the gauge theory/geometry correspondence," hepth/9811131.

[2] H. Ooguri and C. Vafa, "Knot invariants and topological strings," hep-th/9912123, Nucl. Phys. B 577 (2000) 419.

[3] J.M.F. Labastida and M. Mariño, "Polynomial invariants for torus knots and topological strings," hep-th/0004196.

[4] P. Ramadevi and T. Sarkar, "On link invariants and topological string amplitudes," hep-th/0009188.

[5] E. Witten, "Chern-Simons gauge theory as a string theory," hep-th/9207094, in The Floer memorial volume, H. Hofer, C.H. Taubes, A. Weinstein and E. Zehner, eds., Birkhäuser 1995, p. 637.

[6] E. Witten, "Quantum field theory and the Jones polynomial," Commun. Math. Phys. 121 (1989) 351.

[7] R. Gopakumar and C. Vafa, "M-theory and topological strings, I," hep-th/9809187.

[8] C. Faber and R. Pandharipande, "Hodge integrals and Gromov-Witten theory," math.AG/9810173, Invent. Math. 139 (2000) 173.

[9] C. Vafa, "Superstrings and topological strings at large $N$," hep-th/0008142.

[10] R. Gopakumar and C. Vafa, "M-theory and topological strings, II," hep-th/9812127.

[11] W. Fulton and J. Harris, Representation theory. A first course, Springer-Verlag, 1991.

[12] P. Rama Devi, T.R. Govindarajan and R.K. Kaul, "Three-dimensional Chern-Simons theory as a theory of knots and links (III). Compact semisimple group," hepth/9212110, Nucl. Phys. B 402 (1993) 548.

[13] S. Katz, A. Klemm and C. Vafa, "M-theory, topological strings, and spinning blackholes," hep-th/9910181.

[14] W.B.R. Lickorish, An introduction to knot theory, Springer-Verlag, 1998.

[15] W.B.R. Lickorish and K.C. Millett, "A polynomial invariant of oriented links," Topology 26 (1987) 107.

[16] E. Guadagnini, M. Martellini and M. Mintchev, "Wilson lines in Chern-Simons theory and link invariants," Nucl. Phys. B 330 (1990) 575.

[17] M. Álvarez, J.M.F. Labastida, and E. Pérez, "Vassiliev invariants for links from ChernSimons perturbation theory," hep-th/9607030, Nucl. Phys. B 488 (1997) 677.

[18] E. Witten, "Gauge theories and integrable lattice models," Nucl. Phys. B 322 (1989) 629.

[19] J.M. Isidro, J.M.F. Labastida, and A.V. Ramallo, "Polynomials for torus links from Chern-Simons gauge theories," hep-th/9210124, Nucl. Phys. B 398 (1993) 187.

[20] S.G. Naculich and H.J. Schnitzer, "Duality between $S U(N)_{k}$ and $S U(k)_{N}$ WZW models," Nucl. Phys. B 347 (1990) 687. 
[21] S.G. Naculich, H.A. Riggs and H.J. Schnitzer, "Group level duality in WZW models and Chern-Simons theory," Phys. Lett. B 246 (1990) 417. E.J. Mlawer, S.G. Naculich, H.A. Riggs and H.J. Schnitzer, "Group-level duality of WZW coefficients and ChernSimons link observable," Nucl. Phys. B 352 (1991) 863. S.G. Naculich, H.A. Riggs and H.J. Schnitzer, "Simple-current symmetries, rank-level duality, and linear skein relations for Chern-Simons graphs," hep-th/9205082, Nucl. Phys. B 394 (1993) 445.

[22] J.M.F. Labastida, P.M. Llatas and A.V. Ramallo, "Knot operators in Chern-Simons theory," Nucl. Phys. B 348 (1991) 651.

[23] S. Elitzur, G. Moore, A. Schwimmer and N. Seiberg, "Remarks on the canonical quantization of the Chern-Simons-Witten theory," Nucl. Phys. B 326 (1989) 108. J.M.F. Labastida and A.V. Ramallo, "Operator formalism for Chern-Simons theories," Phys. Lett. B 227(1989) 92; "Chern-Simons theory and conformal blocks," Phys. Lett. B 228 (1989) 214. S. Axelrod, S. Della Pietra, and E. Witten, "Geometric quantization of Chern-Simons gauge theory," J. Diff. Geom. 33 (1991) 787.

[24] J.M.F. Labastida and M. Mariño, "The HOMFLY polynomial for torus links from Chern-Simons gauge theory," hep-th/9402093, Int. J. Mod. Phys. A 10 (1995) 1045.

[25] J. Milnor, Singular points of complex hypersurfaces, Annals of Mathematics Studies, No. 61, Princeton University Press, 1968. 